\title{
Transmembrane protein ESDN promotes endothelial VEGF signaling and regulates angiogenesis
}

\author{
Lei Nie,1,2,3 Xiaojia Guo,1,2,3 Leila Esmailzadeh,1,2,3 Jiasheng Zhang,,1,2,3 Abolfazl Asadi,1,2,3 \\ Mark Collinge,2,4 Xuan Li,1,2,3 Jun-Dae Kim,,2 Melissa Woolls, ${ }^{2}$ Suk-Won Jin, ${ }^{2}$ Alexandre Dubrac, ${ }^{2}$ \\ Anne Eichmann, ${ }^{2,5}$ Michael Simons, ${ }^{2}$ Jeffrey R. Bender,2,4 and Mehran M. Sadeghi1,2,3 \\ ${ }^{1}$ Cardiovascular Molecular Imaging Laboratory and ${ }^{2}$ Yale Cardiovascular Research Center, Section of Cardiovascular Medicine, \\ Yale University School of Medicine, New Haven, Connecticut, USA. ${ }^{3}$ VA Connecticut Healthcare System, West Haven, Connecticut, USA. \\ ${ }^{4}$ Raymond and Beverly Sackler Foundation Cardiovascular Laboratory, Yale University School of Medicine, New Haven, Connecticut, USA. \\ ${ }^{5}$ CIRB, Collège de France, INSERM U1050, Paris, France.
}

\begin{abstract}
Aberrant blood vessel formation contributes to a wide variety of pathologies, and factors that regulate angiogenesis are attractive therapeutic targets. Endothelial and smooth muscle cell-derived neuropilin-like protein (ESDN) is a neuropilin-related transmembrane protein expressed in ECs; however, its potential effect on VEGF responses remains undefined. Here, we generated global and EC-specific Esdn knockout mice and demonstrated that ESDN promotes VEGF-induced human and murine EC proliferation and migration. Deletion of Esdn in the mouse interfered with adult and developmental angiogenesis, and knockdown of the Esdn homolog $(d c b l d 2)$ in zebrafish impaired normal vascular development. Loss of ESDN in ECs blunted VEGF responses in vivo and attenuated VEGF-induced VEGFR-2 signaling without altering VEGF receptor or neuropilin expression. Finally, we found that ESDN associates with VEGFR-2 and regulates its complex formation with negative regulators of VEGF signaling, protein tyrosine phosphatases PTP1B and TC-PTP, and VEcadherin. These findings establish ESDN as a regulator of VEGF responses in ECs that acts through a mechanism distinct from neuropilins. As such, ESDN may serve as a therapeutic target for angiogenesis regulation.
\end{abstract}

\section{Introduction}

Angiogenesis is a tightly regulated process during development and growth, and wound healing in adult organisms. Dysregulated angiogenesis, whether inadequate or exaggerated, contributes to a wide variety of pathologies, including ischemic heart and peripheral arterial disease, diabetic retinopathy, and malignancy. This has led to considerable interest in therapeutic interventions to either inhibit or promote angiogenesis. VEGF-A is the key growth factor involved in the regulation of vascular development and vasculature growth. The effects of VEGF-A are primarily mediated through high-affinity binding to VEGF receptor 2 (VEGFR-2), which triggers receptor dimerization and phosphorylation, followed by activation of downstream signaling pathways $(1,2)$. VEGF signaling is a tightly controlled process with multiple layers of regulation ensuring a finely tuned signal. Recent investigations have highlighted the importance of intracellular trafficking and phosphatase-mediated receptor dephosphorylation in regulating signaling of VEGFR-2 and the related VEGFR-3 $(3,4)$.

In the case of VEGF-A $A_{165}$, the main circulating VEGF-A isoform, VEGFR-2 co-receptor neuropilin-1 (Nrp1) plays an important role in regulating its signaling output $(5,6)$. Targeted endothelial Nrp1 deletion in mice results in embryonic lethality with abnormal vascular development, including general peripheral vascular branching deficiency, absence of small and medium-size arteries, and failure to septate the outflow tract, among other defects (7). While the mechanism of these

Conflict of interest: The authors have declared that no conflict of interest exists. Citation for this article: J Clin Invest. 2013;123(12):5082-5097. doi:10.1172/JCI67752. effects has not been fully elucidated, Nrp1 has been shown to be involved in formation of the VEGFR-2 signaling complex (8) and mediation of VEGFR-2 trafficking (9).

Endothelial and smooth muscle cell-derived neuropilin-like protein (ESDN) is a 93 - to $127-\mathrm{kDa}$ transmembrane protein, initially cloned from human coronary artery and highly metastatic lung cancer cells $(10,11)$. ESDN (also called CLCP-1 or DCBLD2) and its homolog, DCBLD1, belong to a novel class of proteins, which, similar to neuropilins, contain both discoidin and CUB (for complement C1r/C1s, Uegf, Bmp1) domains. Other groups as well as ours have previously demonstrated that ESDN is upregulated in remodeling human, rat, and mouse arteries $(10,12)$. The structural similarity of ESDN to neuropilins has raised the possibility that it may regulate the effects of VEGF on ECs.

Here, we demonstrate that ESDN regulates VEGF-induced EC proliferation, migration, and signal transduction and modulates developmental and adult angiogenesis. ESDN achieves these effects by regulating VEGFR-2-VE-cadherin/protein tyrosine phosphatase complex formation. These findings reveal a novel role for ESDN in modulating EC function through regulation of VEGF signaling and suggest that ESDN may serve as a target to regulate angiogenesis.

\section{Results}

ESDN is expressed in human ECs and regulates their VEGF-induced proliferation and migration. We confirmed ESDN expression in HUVECs by flow cytometry (Figure 1A), immunostaining (Figure 1B), quantitative RT-PCR (Figure 1C), and Western blotting (Figure 1D). Immunofluorescence staining demonstrated that in ECs, ESDN localizes in part on the cell membrane (Figure 1B). 
A

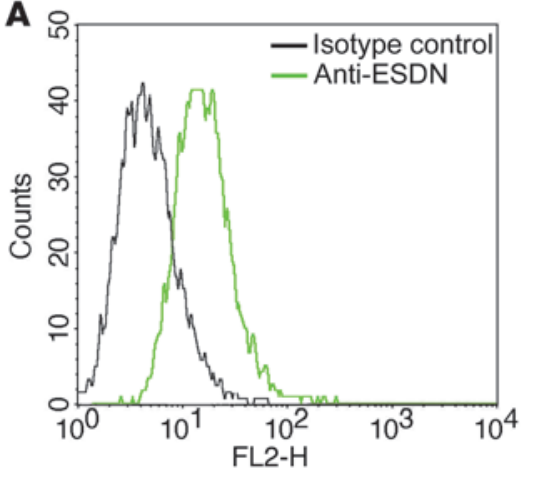

B

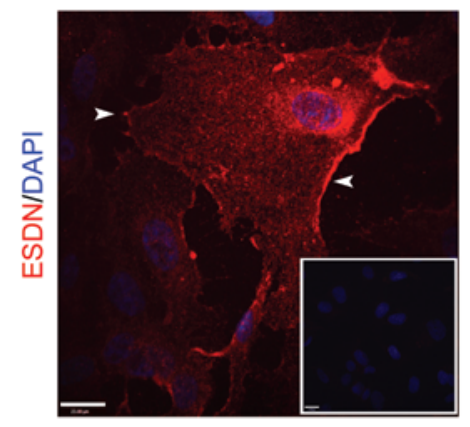

C

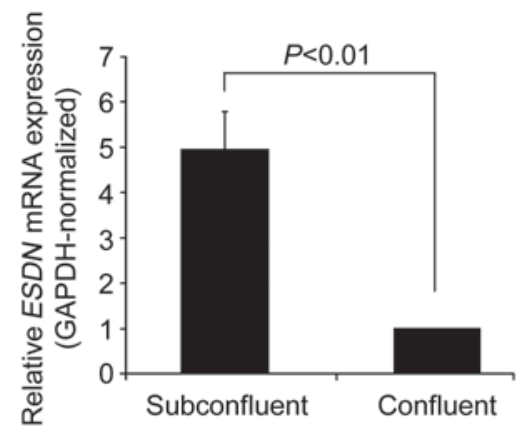

D

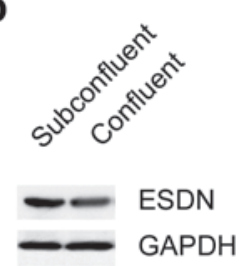

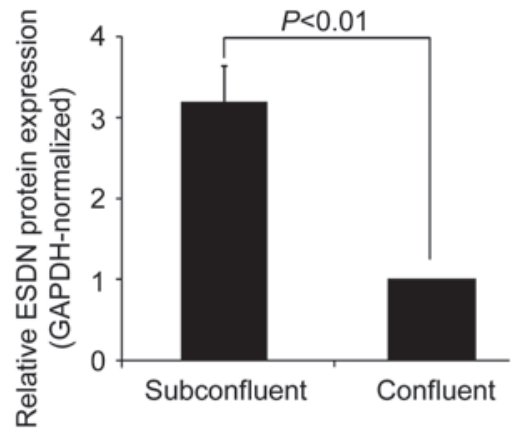

$\mathbf{F}$

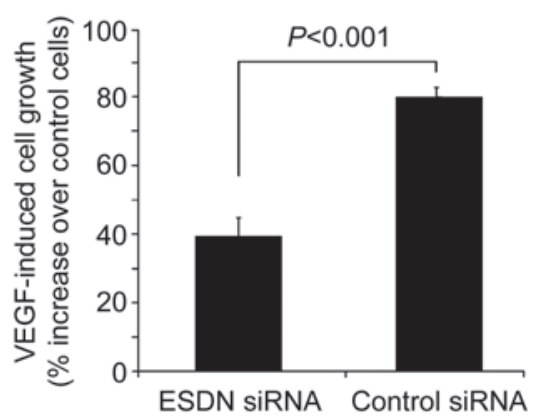

I

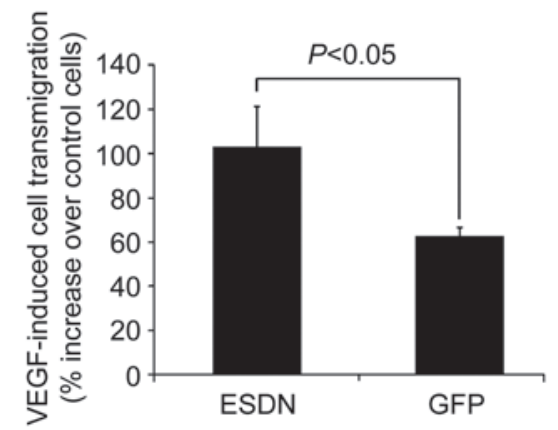

G 드
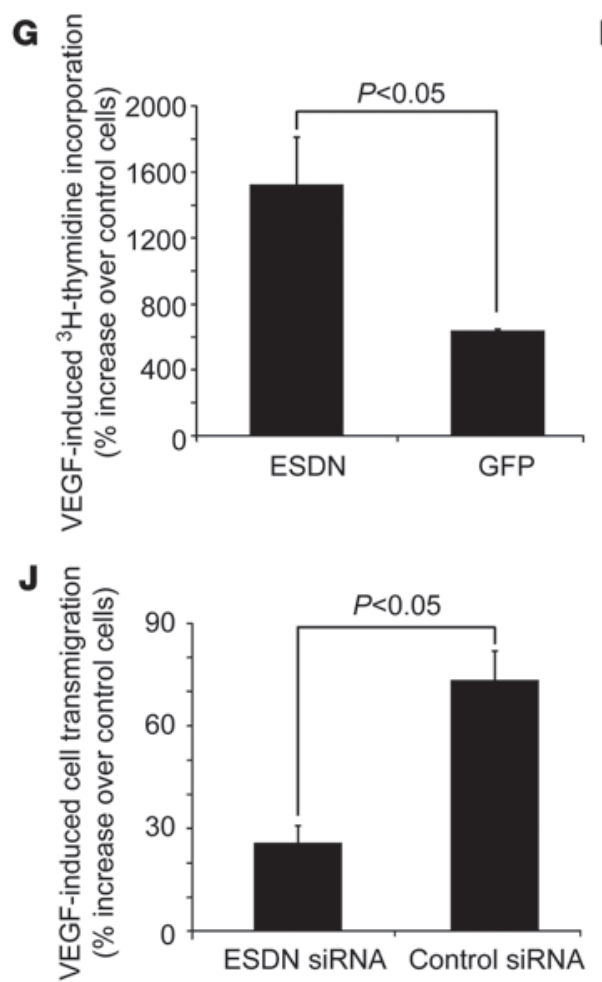

E

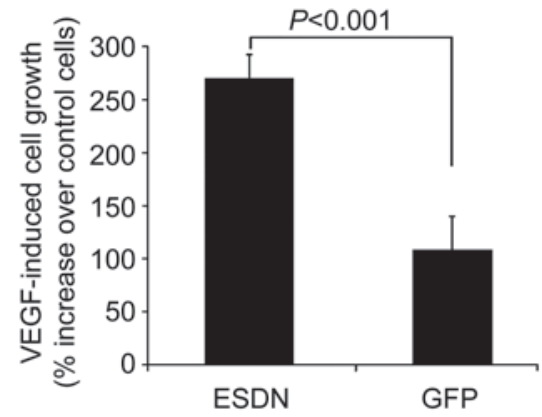

H

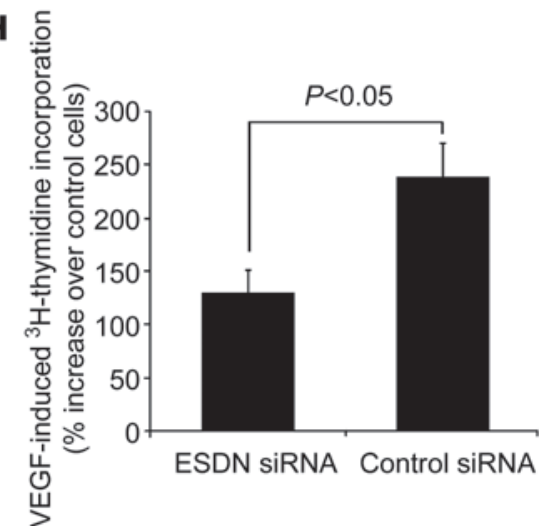

\section{Figure 1}

ESDN modulation of VEGF-induced HUVEC proliferation and migration. (A and B) ESDN flow cytometry and immunofluorescence staining (in red). Arrowheads indicate ESDN expressed on EC membrane. Nuclei are stained with DAPI in blue. Inset: control staining. Scale bar: 20 um. (C and D) GAPDH-normalized ESDN mRNA expression detected by real time RT-PCR $(\mathbf{C}, n=3)$ and ESDN protein expression detected by Western blotting and its quantification $(\mathbf{D}, n=3$ ) in subconfluent and confluent ECs. (E and F) VEGF-induced EC growth monitored through MTT assay following ESDN (or GFP) overexpression (E) or siRNA-mediated ESDN downregulation (F). The data represent percent increase after 4 days relative to non-stimulated cells from three independent experiments. ( $\mathbf{G}$ and $\mathbf{H}$ ) VEGF-induced [ ${ }^{3} \mathrm{H}$-thymidine incorporation following ESDN (or GFP) overexpression (G) or siRNA-mediated ESDN downregulation $(\mathbf{H})$. The data represent percent increase relative to non-stimulated cells from 3 independent experiments. (I and J) VEGF-induced EC Transwell migration following ESDN (or GFP) overexpression (I) or siRNA-mediated ESDN downregulation $(\mathbf{J})$. The data represent percentage increase relative to non-stimulated cells from 3 independent experiments. 
ESDN mRNA (normalized to GAPDH) and protein levels were significantly higher in proliferating, non-confluent ECs compared with growth-arrested, confluent ECs (Figure 1, C and D). The structural similarity of ESDN to neuropilins led us to investigate a potential role for ESDN in regulating VEGF responses in ECs. To this end, we modulated ESDN expression by retroviral transduction and RNA interference in ECs. Compared with ESDN expression in ECs transduced with a control GFPexpressing virus, the ESDN mRNA level increased approximately 3.5-fold in ECs transduced with a HA-tagged ESDN retrovirus (Supplemental Figure 1A; supplemental material available online with this article; doi:10.1172/JCI67752DS1). ESDN-HA expression was confirmed by Western blotting (Supplemental Figure 1B) and immunofluorescence staining, which indicated approximately 90\% transduction efficiency (Supplemental Figure 1C). To downregulate ESDN, we evaluated three siRNA oligonucleotides for their inhibitory effects on ESDN expression in ECs and used the one that decreased ESDN mRNA levels to the greatest degree ( $\sim 50 \%$ of cells transfected with control siRNA) - without affecting the expression of the interferon target gene $2^{\prime}-5^{\prime}$ oligoadenylate synthetase (OAS1) (Supplemental Figure 1D) for the subsequent studies. ESDN protein downregulation following siRNA transfection was confirmed by Western blotting (Supplemental Figure 1E).

Next, we addressed the effect ESDN on EC proliferation by modulating the ESDN expression level. While there was no significant difference in baseline cell numbers in the absence of VEGF between ESDN-modulated and control cells (Supplemental Figure $1 F)$, ESDN overexpression significantly increased (Figure 1E) and ESDN downregulation significantly reduced VEGF-induced cell growth evaluated over a 4-day period (Figure 1F). Similarly, ESDN overexpression significantly increased (Figure 1G) and ESDN downregulation significantly reduced (Figure 1H) VEGF-induced $\left[{ }^{3} \mathrm{H}\right]$-thymidine incorporation. Many regulators of cell proliferation similarly regulate cell migration. Therefore, we explored the potential effect of ESDN on VEGF-induced EC migration. In a modified Boyden chamber migration assay, ESDN overexpression significantly enhanced (Figure 1I) and its downregulation significantly reduced (Figure 1J) VEGF-induced EC transmigration. Together, these data support a role for ESDN in regulating VEGF responses in human ECs.

Generation of Esdn knockout mice. Expression profiling in the mouse showed high levels of ESDN protein in the heart, lungs, and aorta, while it was undetectable in the whole blood (Supplemental Figure 2A). To further investigate the effect of ESDN on VEGF responses in vivo, we knocked out Esdn in mice through homologous recombination (Figure 2A; see Methods for details). We confirmed the deletion of a DNA segment spanning the putative Esdn promoter and exon 1 by Southern blotting (Figure 2B) and the Esdn mRNA knockout by Northern blotting and quantitative RT-PCR using primer sets targeting exons 2-3 and 15-16 (Figure 2, C and D). Es $d n^{-/-}$mice were viable and fertile. En face and cross-sectional immunostaining of adult mouse aortae confirmed the abrogation of ESDN expression, which was mostly localized in the endothelium, in Esdn ${ }^{-/-}$mice (Figure 2E and Supplemental Figure 2B). Finally, ESDN protein was detectable by Western blotting and flow cytometry in murine lung endothelial cells (MLECs) and, to a lesser extent, by flow cytometry in VSMCs and fibroblasts from WT, but not Es $d n^{-/}$, mice (Figure 2, F and G, and Supplemental Figure 2C).
Adult angiogenesis and responses to exogenous VEGF are attenuated in $E s d n^{-/}$mice. To examine the effect of Esdn gene deletion on physiological angiogenesis in adult mice, we used a hindlimb ischemia model. Following ligation of the common femoral artery in 8- to 12-week-old Esdn $n^{--}$and WT control mice, we quantified serial changes in blood flow over time by Laser-Doppler imaging. There was a similar degree of blood flow reduction in the ischemic hindlimb immediately after femoral artery ligation in $E s d^{-/-}$and WT mice. In WT animals, blood flow recovered to baseline levels by 14 days after surgery. However, blood flow recovery was significantly impaired in Es $d n^{-/}$animals (Figure 3, A and B). Both arteriogenesis and distal limb angiogenesis contribute to blood flow restoration in this model. Micro-CT analysis of arteries at 14 days did not show any significant difference in arterial vasculature formation (data not shown). However, histological analysis of the gastrocnemius muscle showed that although there was no difference in distal capillary density, assessed by immunostaining (for CD31) and quantitative RT-PCR (for VE-cadherin) in control limbs, the ligation-induced increase in capillary density was significantly reduced in Esdn $n^{-/-}$ relative to WT animals at 14 days (Figure 3, C-E). This was associated with a parallel reduction in NG2 (pericyte), but not $\alpha$-SMA or PDGFR- $\beta$, staining in $E s d n^{-/}$animals (Supplemental Figure 3, A-F).

Next, we investigated the contribution of ESDN to in vivo responses to exogenous VEGF in adult mice. The effect of $E s d n$ gene deletion on VEGF-induced angiogenesis was assessed in ear angiogenesis and Matrigel assays. While there was no difference in vascular density between $E s d n^{-1}$ and WT mice in control animals, we detected a significant reduction in VEGF-induced ear (Figure 4, A-C) and Matrigel (Figure 4, D-F) angiogenesis assessed by immunostaining (for CD31) and mRNA expression (for VE-cadherin) in Es $d n^{-/}$compared with WT mice. Similar to previous observations in the gastrocnemius muscle, a reduction in pericyte staining, but not $\alpha$-SMA or PDGFR- $\beta$ staining accompanied the angiogenic defect in response to VEGF in the ear and Matrigels (Figure 4, G and H, and Supplemental Figure 3, G-P). Next, we investigated the effect of Esdn gene deletion on upstream VEGF signaling in vivo. Following intravenous administration of VEGF, eNOS phosphorylation, a critical intermediary of VEGF effects in vivo (13), was significantly reduced in the lungs of Esdn $n^{-/-}$mice (Figure 4, I and J), indicating that ESDN is required for proper VEGF signaling in vivo.

Esdn/dcbld2 modulates developmental angiogenesis in mice and zebraf$i s h$. To investigate whether, similar to its effect in adult angiogenesis, ESDN modulates developmental angiogenesis in mice, retinal blood vessels were compared between Es $d n^{-1-}$ mice and their WT littermates. On postnatal day 5 there was a significant reduction in the total length of blood vessels and number of branches detected by isolectin immunostaining in $E s d n^{-/-}$animals (Figure 5, A-C). The angiogenic defect in $E s d n^{-/}$retinas was reflected in a similar reduction in the pericyte network (Figure 5, D and E). However, Esdn gene deletion had no significant effect on VSMCs, as reflected in $\alpha$-SMA and PDGFR- $\beta$ staining of the retinas (Supplemental Figure 4, A-D). By postnatal day 10, with progression of retinal angiogenesis, the retina was entirely covered with blood vessels in Es $d n^{-/-}$and WT mice (data not shown). Given the central role of VEGF in postnatal retinal angiogenesis (14), these results imply a role for ESDN in regulating VEGF responses in vivo.

To address the contribution of EC ESDN to the observed effect of $E s d n$ gene deletion on retinal angiogenesis, we generated EC-specific Esdn knockout mice by crossing transgenic mice expressing Cre recombinase under the control of the VE-cadherin promoter (15) 
$\mathbf{A}$
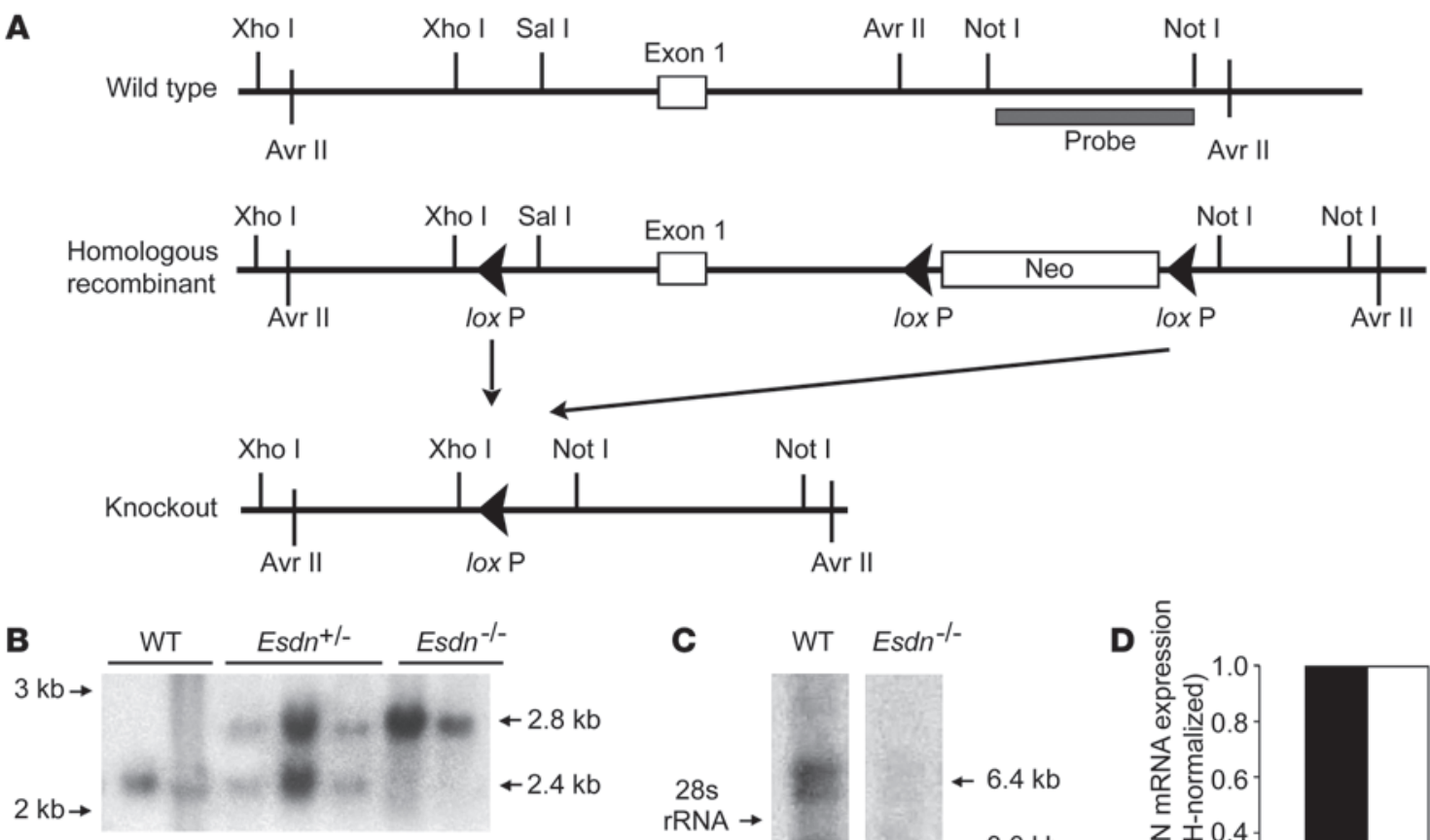

$\leftarrow 2.8 \mathrm{~kb}$

$\leftarrow 2.4 \mathrm{~kb}$

E
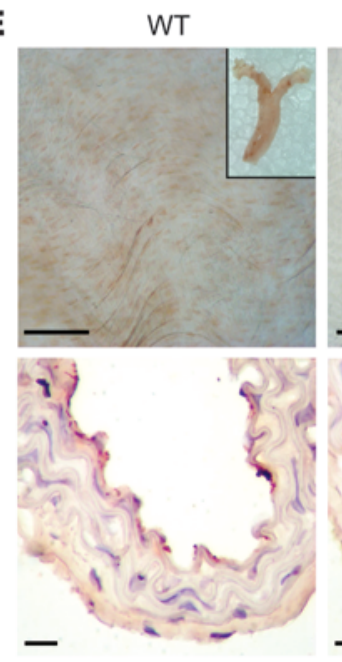

$E s d n^{-/-}$
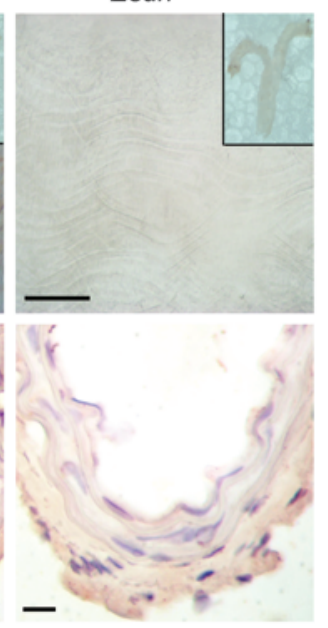

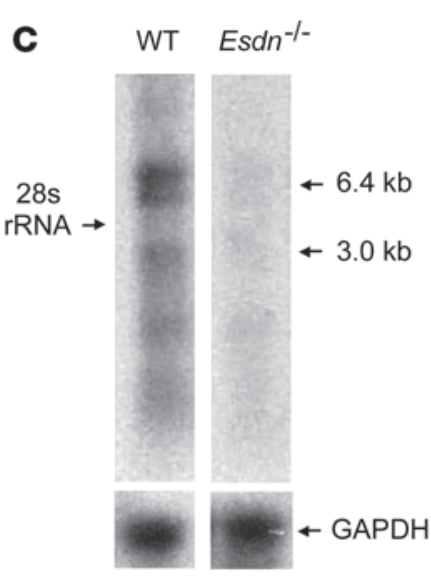

F

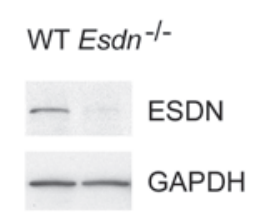

D $\frac{.}{y}$

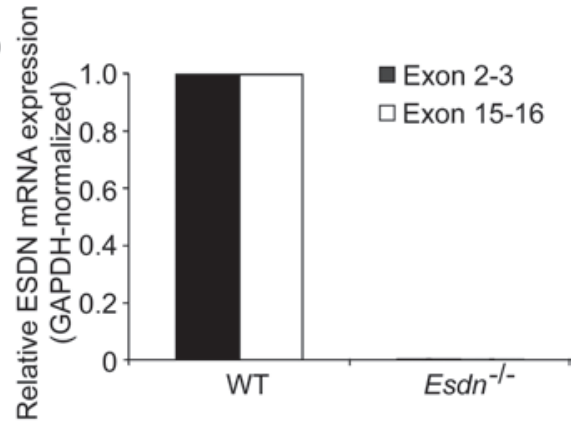

G
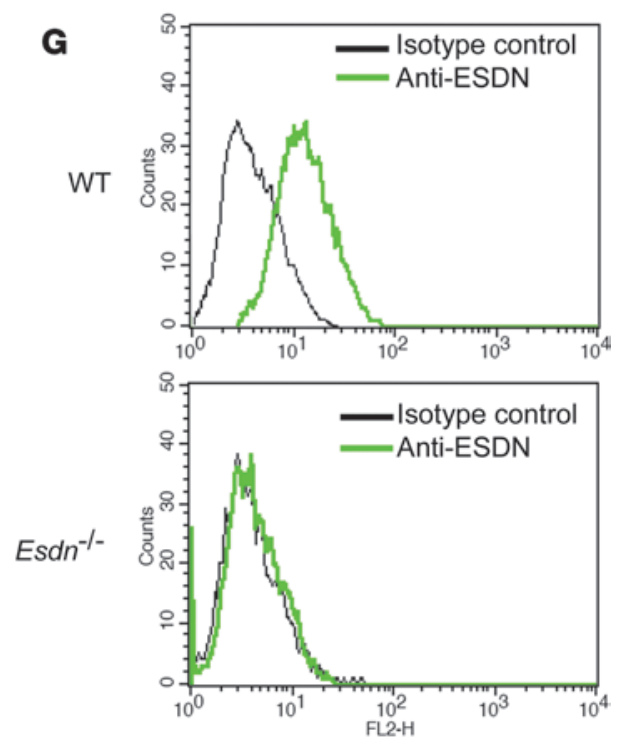

Figure 2

Generation of Esdn knockout mice. (A) Schematic diagram of the strategy used to generate the Esdn ${ }^{-1-}$ mouse. The location of the 3' probe for Southern blotting is shown. (B) Southern blot analysis of genomic DNA from WT, heterozygous (Esdn+/-), and $E s d n^{-1-}$ mice digested with Avr II. (C and D) Northern blotting (C) and real time RT-PCR (D) to assess Esdn mRNA expression in the WT and Esdn ${ }^{-/-}$mice. The two primer pairs span exons 2 and 3 and exons 15 and 16 of Esdn. (E) En face (top row) and cross-sectional (lower row) ESDN immunostaining of aortas from WT and $E s d n^{-/-}$mice. Insets show low magnification images of proximal aorta. Scale bars: $100 \mu \mathrm{m}$. (F and $\mathbf{G}$ ) Western blotting and flow cytometric analysis of ESDN expression in MLECs from WT and Esdn ${ }^{-/}$mice.

and heterozygous for Esdn (VE-cadherin $\left.\mathrm{Cre}^{+/-}, E s d n^{+/}\right)$with $E s d n^{f / f l}$

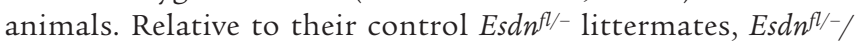
VE-cadherin Cre mice had a significant reduction in the total length of retinal blood vessels and number of branches on postnatal day 5 (Figure 5, F-H), indicating the involvement of EC ESDN in the regulation of retinal angiogenesis. Incomplete deletion or a potential involvement of other sources of ESDN may have contributed to the less severe phenotype observed in EC-specific Esdn knockout mice. As in global Esdn $n^{--}$animals, pericyte staining was reduced in EC-specific knockout mice (Figure 5, I and J). 

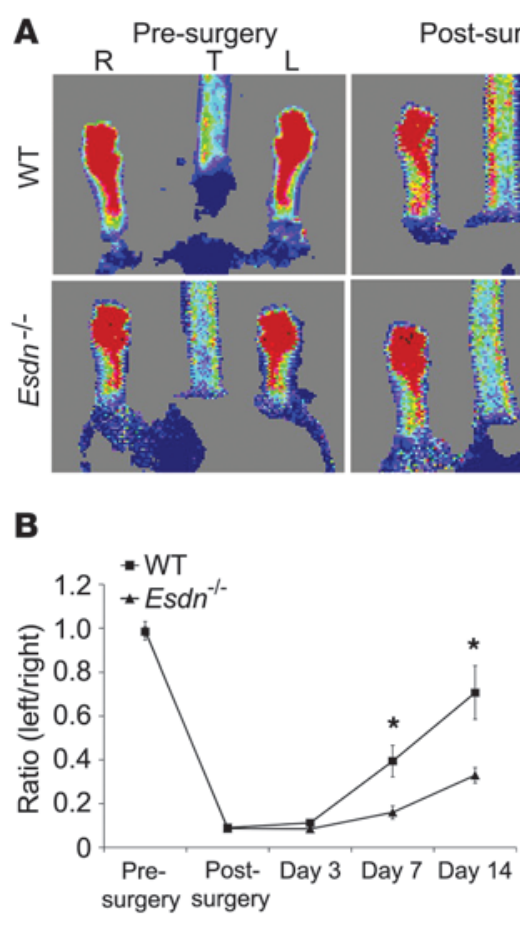

Figure 3

Attenuation of adult angiogenesis in Esdn ${ }^{-/-}$mice. (A-E) Hindlimb ischemia-induced neovascularization. Representative examples of laserDoppler images $(\mathbf{A})$ and quantification of hindlimb blood flow $(\mathbf{B})$ before and after left femoral artery ligation. ${ }^{*} P<0.05 . n=3$. Representative examples (C) and quantification of CD31 immunostaining of calf blood vessels $(\mathbf{D}, n=6)$ and calf muscle GAPDH-normalized VE-cadherin mRNA expression $(\mathbf{E}, n=3)$ at 14 days after femoral artery ligation in WT or Esdn $n^{-/-}$mice. Scale bars: $100 \mu \mathrm{m}$.

The vasculature of zebrafish embryos at early developmental stage is devoid of any supporting cells, including mural cells and pericytes and only contains ECs $(16,17)$. To further investigate the effect of ESDN/DCBLD2 in sprouting angiogenesis, we examined whether esdn/dcbld2 is expressed in zebrafish ECs and whether its knockdown affects vascular development in this organism. Expression of esdn/dcbld2 was confirmed by RT-PCR in flk+ zebrafish ECs (Supplemental Figure 4E), and morpholinos (MOs) that selectively target the $d c b l d 2$ splicing junction between intron 1 and exon 2 (sp1) or exon 2 and intron $2(\mathrm{sp} 2$ ) significantly reduced the number of intersegmental vessels (ISVs) sprouting from the dorsal aorta in injected embryos (Figure 5, K and L, Supplemental Figure 4F, and data not shown). Moreover, the injected embryos lacked the majority of dorsal anastomosis longitudinal vessels (DLAVs), which are formed by anastomosis of ISVs, as the remaining ISVs failed to reach the dorsal-most region of the embryos (Figure $5 \mathrm{~K}$ ). Of note, gross morphology was unaffected by dcbld $2 \mathrm{MO}$ injection (Figure $5 \mathrm{~K}$ ), there was no pronounced cardiac edema in injected embryos, and the heart rate was comparable to that of control embryos (Sup- plemental Videos 1 and 2). Considering that VEGF-A is the master regulator of sprouting angiogenesis in zebrafish and the morphogenesis of ISV is largely dependent on VEGF-A signaling (18, 19), the phenotype of $d c b l d 2 \mathrm{MO}$-injected embryos suggested that ESDN/dcbld2 may modulate VEGF-A signaling in development.

ESDN modulates VEGF signaling in ECs. Similar to our observations in human ECs, VEGF-induced cell proliferation (Supplemental Figure 5A), $\left[{ }^{3} \mathrm{H}\right]$-thymidine incorporation (Supplemental Figure 5B), and Transwell migration (Supplemental Figure 5, C and D) were attenuated in Esdn ${ }^{-/-}$MLECs. To elucidate the mechanism(s) of the observed effects of ESDN on VEGF responses in ECs, we assessed the effect of Esdn downregulation or gene deletion on upstream VEGF signaling events. Western blot analysis of WT or Esdn $n^{-1-}$ MLECs exposed to VEGF demonstrated reduced activation of p44/42 and p38 MAPK and reduced Akt activation in Esdn $n^{-/-}$cells (Figure 6, A-D). Transient transfection of ESDN in Esdn $n^{-/-}$MLECs (which is limited to a subset of cells) partially reversed the inhibitory effect of gene deletion on VEGF-induced MAPK p44/42 phosphorylation (Figure 6, E-G). The difference 

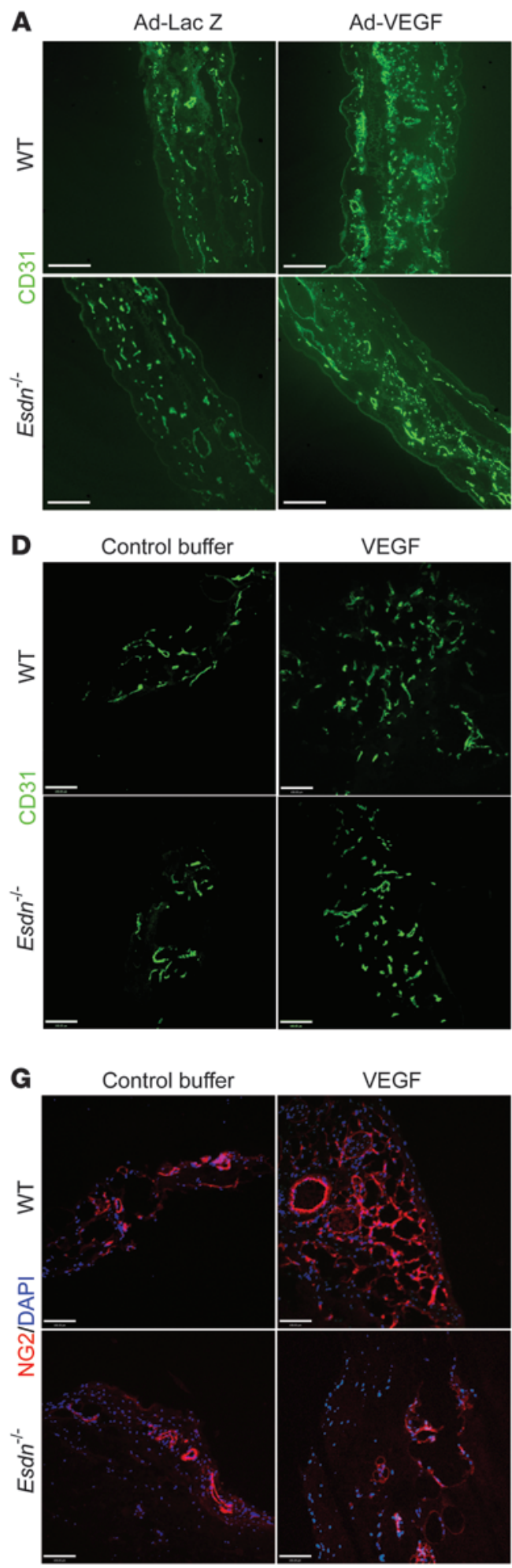

Figure 4

Attenuation of responses to exogenous VEGF in Esdn ${ }^{-/-}$mice.(A-C) Ear angiogenesis. Examples (A) and quantification of CD31 immunostaining of ear blood vessels (B, $n=6$ ) and ear GAPDH-normalized VE-cadherin mRNA expression $(\mathbf{C}, n=3) 5$ days following intradermal injection of AdVEGF or Ad-LacZ into opposite ears of WT and Esdn ${ }^{-1-}$ mice. Scale bars: $100 \mu \mathrm{m}$. (D-H) Matrigel angiogenesis. Examples (D) and quantification of CD31 immunostaining (E, $n=6)$ and VE-cadherin mRNA $(\mathbf{F}, n=3)$ of Matrigel plugs containing VEGF or control buffer implanted in WT and Esdn ${ }^{-/-}$mice. Scale bars: $100 \mu \mathrm{m}$. (G and $\left.\mathbf{H}\right)$ Representative examples $(\mathbf{G})$ and quantification $(\mathbf{H})$ of NG2 pericyte immunostaining of Matrigels implanted in WT and Esd $n^{-1-}$ mice. Nuclei are stained with DAPI in blue. $n=3$. Scale bar: $100 \mu \mathrm{m}$. (I and J) In vivo VEGF signaling. Example (I) and quantification $(\mathbf{J}, n=3)$ of VEGF-induced lung eNOS phosphorylation in WT and $E s d n^{-1-}$ mice. 


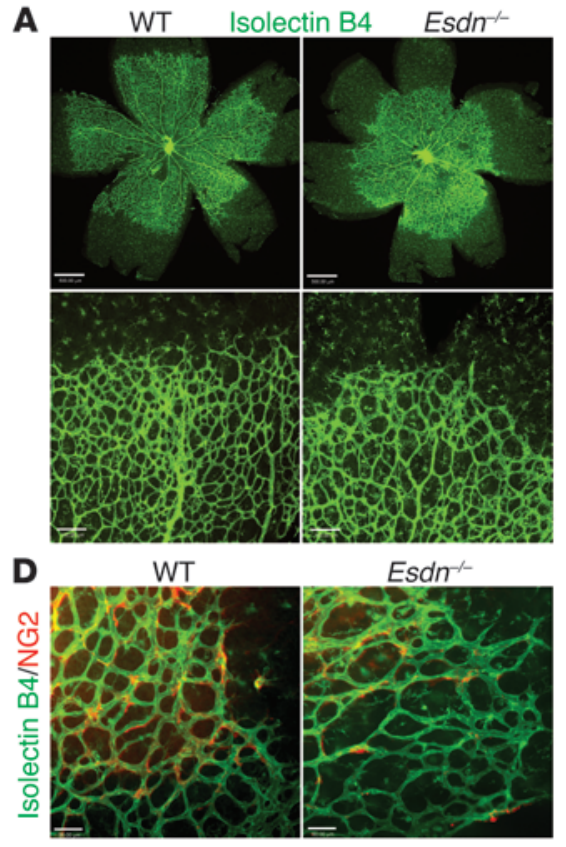

F Control Isolectin B4 Esdn ${ }^{\triangle E C}$
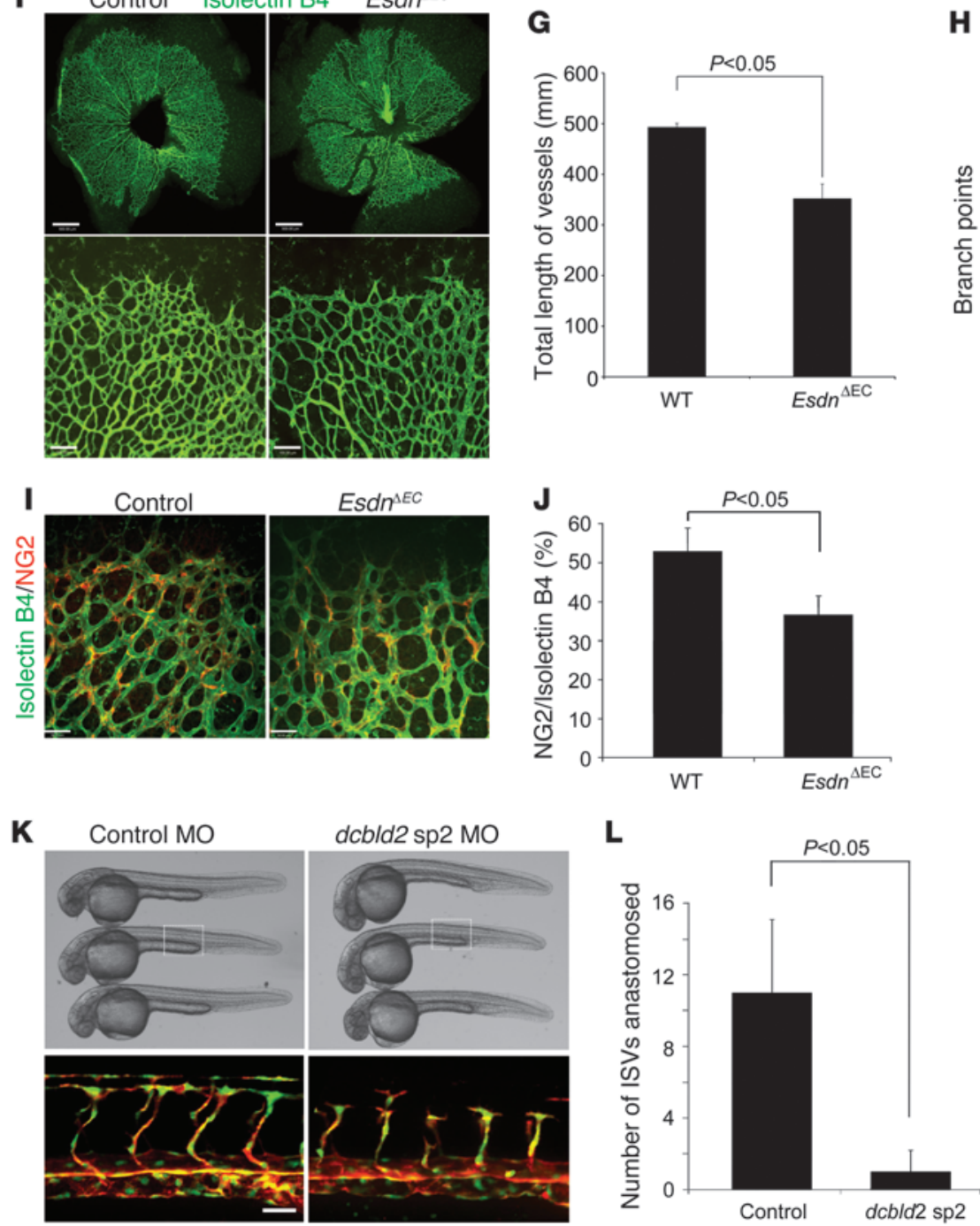


\section{Figure 5}

ESDN/dcbld2 modulates developmental angiogenesis in mice and zebrafish. (A-E) Retinal angiogenesis in $E s d n^{-/}$mice. Representative examples $(\mathbf{A})$ and quantification in the entire retina (B and $\mathbf{C}$ ) of isolectin staining of blood vessels in WT and $E s d n^{-1-}$ mice on postnatal day 5 . $n=12$. Scale bars: $500 \mu \mathrm{m}$, upper panels; $100 \mu \mathrm{m}$, lower panels. (D and E) Retinal pericyte staining. Representative examples (D) and quantification (E) of NG2 immunostaining of retinal blood vessels in WT and $E s d n^{-1-}$ mice on postnatal day 5. $n=6$. Scale bars: $50 \mu \mathrm{m}$. $(\mathbf{F}-\mathbf{J})$ Retinal angiogenesis in EC-specific Esdn knockout mice. Representative examples $(\mathbf{F})$ and quantification in the entire retina $(\mathbf{G}$ and $\mathbf{H})$ of isolectin staining of blood vessels in Esd ${ }^{f l-} / \mathrm{VE}$-cadherin Cre mice and their control $E s d n^{f l-}$ littermates on postnatal day 5. $n=8$. Scale bars: $500 \mu \mathrm{m}$ upper panels, $100 \mu \mathrm{m}$ lower panels. (I and J) Retinal pericyte staining. Representative examples (I) and quantification ( $\mathbf{J})$ of NG2 immunostaining of retinal blood vessels in Esdn tll-/VE-cadherin

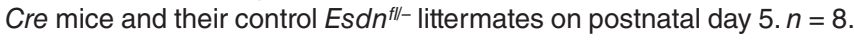
Scale bars: $50 \mu \mathrm{m}$. (K and $\mathbf{L}$ ) Vascular development in zebrafish. (K) Brightfield (upper panels) and epifluorescence (lower panels) images of $28 \mathrm{hpf} T g(f l i 1: n E G F P) ; T g(k d r l: m$ Cherry) zebrafish embryos (head to the left) injected with control or dcbld2/esdn sp2 MO. In embryos injected with dcbld2MO, intersegmental vessels often consist of slender endothelial cells and/or are stalled along their dorsal trajectory. Nuclei of fli 1-positive cells are shown in green and $k d r l$-positive cells in red. Scale bar: $25 \mu \mathrm{m}$. (L) Quantification of the number of ISVs anastomosed per embryo ( $n=20$ in each group).

in VEGF signaling between $E s d n^{-/-}$and $E s d n^{+/+}$MLECs persisted in the presence of increasing amounts of VEGF (Figure 6, H-J). This differential downstream VEGFR-2 signaling was also present when VEGF-A $A_{121}$ rather than VEGF-A 165 $_{\text {was }}$ was to stimulate ECs (Supplemental Figure 6, A-F). Similar to the effect of Esdn gene deletion in murine ECs, siRNA-mediated Esdn knockdown attenuated VEGF-induced p44/42 and p38 MAPK phosphorylation in HUVECs (Supplemental Figure 6, G-I).

To determine the reason for the reduced downstream VEGFR-2 signaling in $E s d n^{-/-}$ECs, we next examined the state of VEGFR-2 phosphorylation after VEGF treatment. siRNA-mediated downregulation of ESDN significantly reduced VEGF-induced VEGFR-2 Tyr1054/1059, Tyr1175, and Tyr1214 phosphorylation in HUVECs (Figure 7, A and B). A similar effect of ESDN on VEGFR-2 phosphorylation was observed in MLECs (Supplemental Figure 7, $A$ and $B$ ), indicating that the absence of ESDN impairs VEGF signaling in ECs.

The reduced activation of VEGFR-2 in the absence of ESDN may have resulted from changes in the expression of various regulators of VEGF signaling, such as Nrp1 or VEGFR-1, or VEGFR-2 itself (20). ESDN downregulation did not alter VEGFR-1 and VEGFR-2 mRNA or protein expression in human and murine ECs (Supplemental Figure 7, C and D). Similarly, ESDN downregulation did not affect Nrp1-1 or Nrp1-2 expression in ECs (Supplemental Figure $7 E$ ). Next we investigated whether ESDN regulates cell surface VEGFR-1 or VEGFR-2 expression. Similar to its lack of effect on total VEGFR expression, ESDN downregulation did not alter cell surface VEGFR-1 or VEGFR-2 expression, detected by flow cytometry (Supplemental Figure 7F), indicating that ESDN alters VEGF signaling in ECs, independent of the VEGFR expression level.

We next sought to determine whether ESDN associates with VEGFR-2. ESDN immunoprecipitation followed by immunoblotting for VEGFR-2 and VEGFR-2 immunoprecipitation and then ESDN immunoblotting confirmed that ESDN and VEGFR-2 associate in a complex in human ECs (Figure 7C).
Interestingly, we did not detect any consistent effect of VEGF stimulation on this association (data not shown), indicating that the ESDN-VEGFR-2 association was not dependent on VEGFR-2 phosphorylation.

Association of VEGFR-2-phosphatase and VEGFR-2-VE-cadherin is regulated by ESDN. VEGFR-2 signaling is modulated through its association with a number of key regulatory proteins, including certain protein tyrosine phosphatases, such as proteintyrosine phosphatase 1B (PTP1B) $(4,21)$, T cell protein tyrosine phosphatase (TCPTP) (22), and vascular endothelial protein tyrosine phosphatase (VE-PTP) $(23,24)$, among others. The profound effect of ESDN on downstream VEGF signaling and its association with VEGFR-2 led us to investigate whether ESDN modulates regulatory macromolecular complexes involving VEGFR-2, protein phosphatases, and VE-cadherin. In MLECs, VEGFR-2 immunoprecipitation followed by immunoblotting for PTP1B and TCPTP demonstrated enhanced phosphatase co-immunoprecipitation in Esdn ${ }^{-/-}$cells compared with $E s d n^{+/+}$ cells (Figure 7D). Importantly, Esdn gene deletion had no effect on PTP1B or TCPTP expression in MLECs (Figure 7E and Supplemental Figure 7G).

VE-cadherin regulates VEGF signaling and PTP1B stabilizes VE-cadherin on the EC membrane at sites of cell-cell contact (21). Therefore, we investigated whether ESDN modulates VEGFR-2VE-cadherin association in MLECs. Similar to the effect of Esdn gene deletion on VEGFR-2-phosphatase association, VEGFR-2 immunoprecipitation followed by immunoblotting for VE-cadherin demonstrated enhanced VEGFR-2-VE-cadherin co-immunoprecipitation in Esdn $n^{-/}$MLECs (Figure 7D). Again, Esdn gene deletion had no effect on VE-cadherin expression (Figure 7E and Supplemental Figure 7G).

To further address the role of these tyrosine phosphatases in the modulatory effect of ESDN on VEGF signaling, we used siRNA

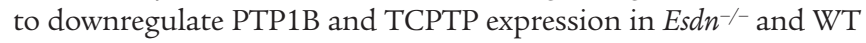
MLECs and quantified VEGF-induced P44/42 MAPK phosphorylation. A similar degree of phosphatase downregulation was obtained in $E s d n^{-/-}$and $E s d n^{+/+}$cells (Supplemental Figure 7, H-M). Consistent with previous reports, phosphatase downregulation did not alter VEGF-induced p44/42 MAPK phosphorylation in Es $d n^{+/+}$cells (4). In $E s d n^{-/}$cells, the siRNA-mediated PTP1B or TCPTP downregulation significantly enhanced VEGF-induced p44/42 MAPK and Akt phosphorylation (Figure 8). Similarly, phosphatase downregulation restored VEGFR-2 phosphorylation in Es $d n^{-/}$MLECs (Figure 8). The combined downregulation of PTP1B and TCPTP enhanced VEGF signaling to a greater degree than downregulation using either siRNA alone (Supplemental Figure 7, N-P).

\section{Discussion}

Here we identified ESDN as a novel regulator of developmental and adult angiogenesis, demonstrating that it modulates VEGF signaling by preventing VEGFR-2 complex formation with its negative regulators, $\mathrm{VE}$-cadherin and the protein tyrosine phosphatases PTP-1B and TC-PTP (Figure 9). Members of the VEGF family of glycoproteins interact with three receptor tyrosine kinase (RTK) VEGFRs. VEGFR-1 and -2 are both expressed on ECs and bind to VEGF-A, while VEGFR-3 is a receptor for VEGF-C and VEGF-D and has a more limited endothelial expression pattern in adults (20). Despite the higher affinity of VEGF for VEGFR-1, VEGFR-2 is the major mediator of the mitogenic, angiogenic, and permeability-enhancing effects of VEGF in ECs. VEGF binding to 
A

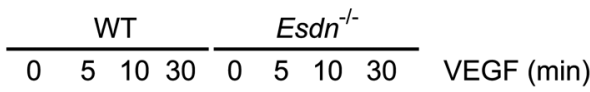

$\begin{array}{ll}=-==-= & \text { P-MAPK p44/42 } \\ =-m= & =\text { Total MPAK p44/p42 }\end{array}$

- $\quad$ P-p38

$\ldots \ldots \ldots$ Total p38

m $-\ldots$ P-Akt
E

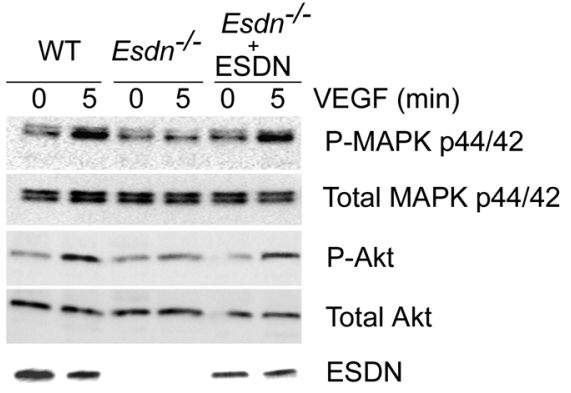

B

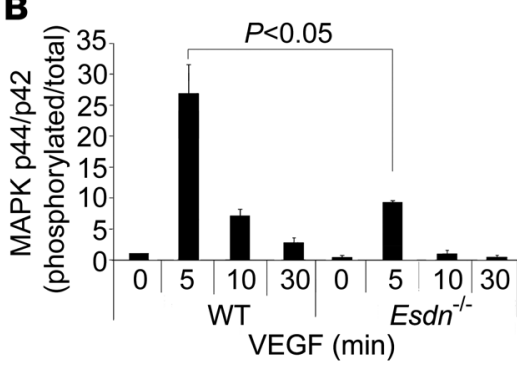

C

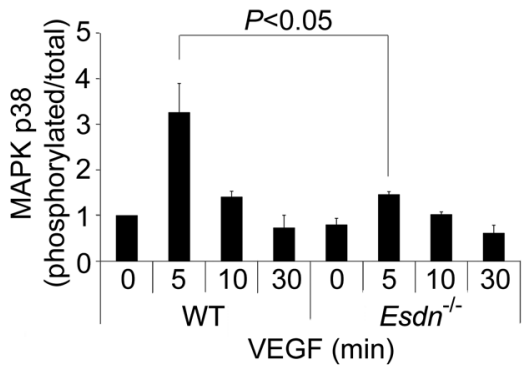

D

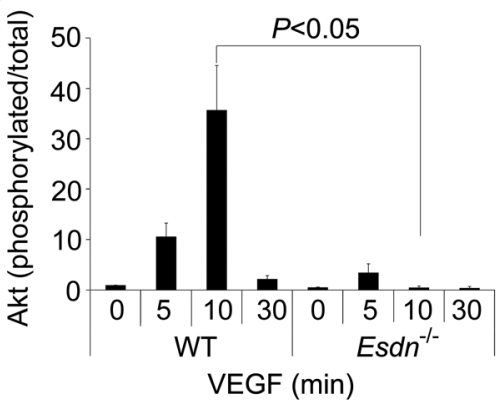

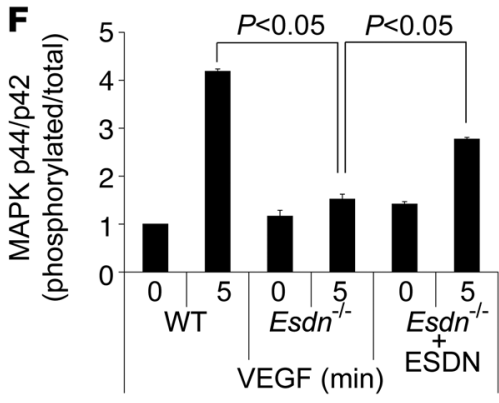

G

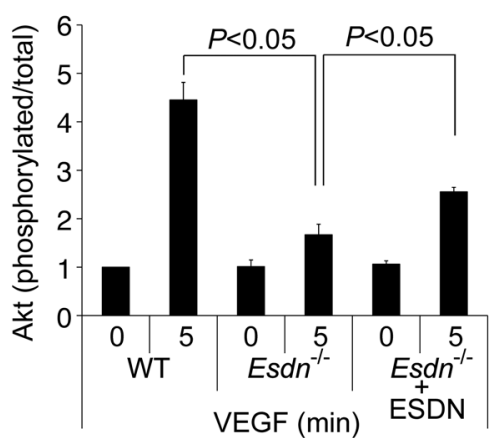

H

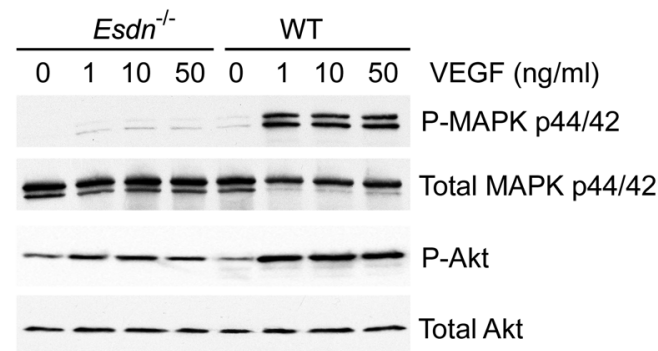

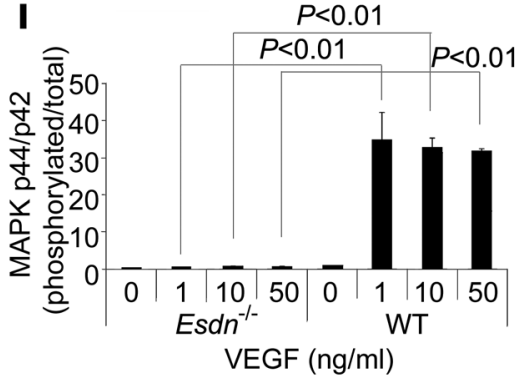

J

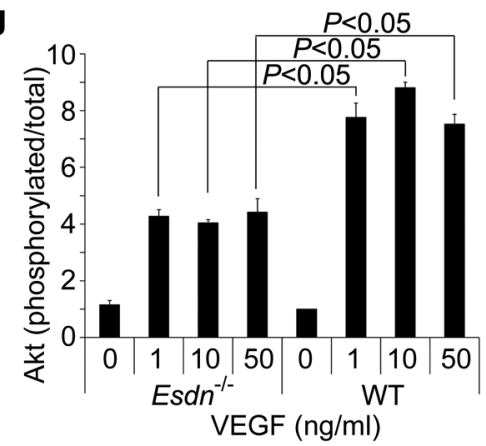

Figure 6

Attenuation of VEGF signaling in Esdn ${ }^{-/}$MLECs. (A-D) Western blot analysis of VEGF signaling in MLECs. Examples and quantification of VEGFinduced MAPK p44/42 Thr202/Tyr204 phosphorylation (A and B), p38 Thr180/Tyr182 phosphorylation (A and C), and Akt Ser473 phosphorylation $(\mathbf{A}$ and $\mathbf{D})$ in WT and Esdn $n^{-/-}$MLECs. $n=3$. (E-G) ESDN reconstitution in Esdn ${ }^{-1-}$ MLECs. Example (E) and quantification $(\mathbf{F}$ and $\mathbf{G})$ of Western blots of VEGF-induced MAPK p44/p42 Thr202/Tyr204 phosphorylation and Akt Ser473 phosphorylation following transient transfection of Esdn in Esdn ${ }^{-1-}$ MLECs. (H-J) Western blot analysis of MAPK p44/42 Thr202/Tyr204 phosphorylation and Akt Ser473 phosphorylation in WT and $E s d n^{-1-}$ MLECs following stimulation with various concentrations of VEGF.

VEGFR-2 triggers receptor dimerization, transphosphorylation, endocytosis, and the activation of downstream signaling pathways, including Akt and p44/42 and p38 MAPK, which regulate EC survival, proliferation, and migration $(1,2,20)$.
The effects of VEGF in ECs are finely regulated through positive and negative regulators of VEGFR-2 signaling. These regulators include VEGFR-1, which may serve as a "decoy" receptor and negatively regulate VEGF signaling by preventing VEGF binding to 
VEGFR-2. Other regulators of VEGFR-2 signaling control its trafficking and intracellular localization. As for other RTKs, VEGFR-2 internalization and localization in the endosomal compartment play a key role in regulating VEGF signaling, and the inhibition of receptor internalization can lead to reduced receptor-mediated signaling $(4,20,25)$. Ephrin B2, a transmembrane ligand for Eph receptors, interacts with VEGFR-2 and is required for VEGFinduced receptor internalization and proper signaling (3). Activated VEGFR-2 is de-phosphorylated by several phosphotyrosine phosphatases, including PTP1B (21), TC-PTP (22), VE-PTP (23, 24), DEP-1/CD148 $(26,27)$, and Src-homology phosphatase-1 (SHP1) (28) and SHP2 (29). Phosphatase action interferes with VEGFR-2 internalization and attenuates downstream signaling. Neuropilins are VEGF co-receptors without intrinsic catalytic activity that also bind to and enhance the affinity of VEGFR-2 for $\operatorname{VEGF}(5,6,30)$. Nrp1 was originally identified as a receptor that mediates the chemorepulsant activity of class 3 semaphorins (31). Subsequent work has demonstrated that $\mathrm{Nrp}-1$ is an isoformspecific receptor for VEGF that binds to the exon-7-encoded domain of $\mathrm{VEGF}_{165}$ and enhances VEGF 165 binding to VEGFR-2 and the resultant VEGFR-2 signaling (6). With single CUB, LCCL, and coagulation factor V/VIII (discoidin) domains, the structure of ESDN resembles that of neuropilins, which contain two CUB domains, two coagulation factor V/VIII domains, and a single MAM domain $(10,11,32)$. However, unlike Nrp1 gene deletion, which results in embryonic lethality with abnormal vascular development $(5,6), E s d n^{-/-}$mice are viable, without any major vascular defect as adults. Indeed, we did not detect any significant difference in the size distribution of postnatal day 7 renal and adult hindlimb arteries by ex vivo CT angiography between $E s d n^{-/-}$mice and their WT controls (data not shown). In zebrafish embryos, esdn/dcbld2 downregulation led to defective vascular development. A similar effect on developmental (and adult) angiogenesis was observed in Esdn $n^{--}$mice, indicating regulatory mechanisms of ESDN in the vasculature are distinct from those involving Nrp-1.

ESDN downregulation inhibits VEGF-induced EC proliferation and migration, and ESDN overexpression has the opposite effect. We traced these effects to the modulatory effect of ESDN on VEGF-induced Akt and p42/44 and p38 MAPK phosphorylation and upstream VEGFR-2 phosphorylation. VEGF-induced Akt and $\mathrm{p} 44 / 42$ MAPK activation supports EC survival and proliferation, whereas p38 MAPK phosphorylation mediates actin reorganization and cell migration (20). eNOS phosphorylation, which mediates many VEGF functions, is similarly Akt dependent (20). VEGF binding to VEGFR-2 leads to the phosphorylation of several tyrosine residues, including Tyr1054/1059, which regulates receptor kinase activity; Tyr1175, which supports AKT and MAPK p44/42 phosphorylation and the resultant cell survival and proliferation; and Tyr1214, which supports p38 MAPK phosphorylation and cell migration $(1,20)$. The modulation of ESDN expression altered VEGFR-2 phosphorylation at multiple sites, but had no effect on total VEGF receptor or co-receptor levels or cell membrane VEGFR expression in ECs. ESDN-VEGFR-2 co-immunoprecipitation indicates that ESDN and VEGFR-2 either directly interact or are part of a larger signaling complex. VEGFR-2 is in complex with VE cadherin on the cell membrane (33). It has been reported that the association of VEGFR- 2 with the VE-cadherin- $\beta$-catenin complex and the resultant proximity to CD148 (DEP-1) prevents VEGFR-2 phosphorylation, internalization, and signaling (26). The enhanced VEGFR-2-VE-cadherin association observed in
$E s d n^{-/}$ECs can therefore contribute to the inhibitory effect of Esdn gene deletion on VEGF signaling. Similar to VE-cadherin, Nrp-1 associates with VEGFR-2 and regulates VEGFR-2 endocytosis (2). Interestingly, while VEGFR-2-Nrp-1 complexes are formed when VEGF-A $A_{165}$ simultaneously binds to both receptors (34), VEGF is not required for ESDN-VEGFR-2 co-immunoprecipitation, again indicating the presence of a distinct mechanism for their interaction. Unlike Nrp-1, ESDN has a long cytoplasmic domain with several $\mathrm{SH} 2$ and $\mathrm{SH} 3$ binding sites, which may be involved in interaction with other molecules.

Esdn gene deletion enhanced VEGFR-2 association with PTP1B, TC-PTP, and VE-PTP (data not shown). These phosphatases dephosphorylate the phosphorylation sites (Tyr1054/1059, Tyr1175, and Tyr1214) that we showed to be affected by modulation of ESDN expression. Downregulation of either phosphatase

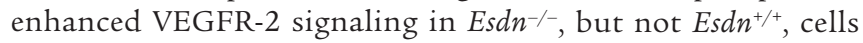
indicating their involvement in the observed effect of ESDN on VEGF signaling. While we focused our studies on these representative phosphatases, we cannot rule out that ESDN may modulate VEGFR-2 association with other phosphatases. PTP1B is a cytoplasmic phosphatase that predominantly resides in the endoplasmic reticulum and regulates RTK activation in the endocytic compartment $(4,35)$. However, PTP1B appears also to have access to activated RTKs at the cell membrane, potentially via peripheral endoplasmic reticulum membranes (35). Interestingly, at least in the case of VEGFR-2, PTP1B association with the kinase domain is independent of the phosphorylation status of the receptor (21). The closely related TC-PTP resides in the cytoplasm and at the plasma membrane and binds to VEGFR-2 independent of exogenous VEGF stimulation and prevents its activation (22). Thus, ESDN can regulate VEGFR-2 signaling by interfering in its association with VE-cadherin and PTPs.

Similar to other RTKs, VEGFR-2 undergoes constant recycling between the cell membrane and intracellular pools $(4,33,36)$. Ligand-induced activation of RTKs leads to receptor ubiquitination and targeting to lysosomal machinery for degradation (37). Contrary to its effect on VEGF responses in ECs, ESDN downregulation promotes PDGF-induced VSMC proliferation and migration. The effect of ESDN downregulation in VSMCs is linked to enhanced cell membrane PDGFR- $\beta$ levels and the inhibition of PDGFR- $\beta$ ubiquitination and lysosomal degradation through regulation of ubiquitin ligase c-Cbl expression (38). c-Cbl is also involved in VEGFR-2 ubiquitination and degradation $(39,40)$. While we did not detect any difference in c-Cbl expression between Esdn ${ }^{-/}$and WT MLECs (data not shown), a similar involvement of $\mathrm{c}-\mathrm{Cbl}$ in mediating the effects of ESDN downregulation on VEGFR-2 signaling in ECs would be expected to result in enhanced, rather than attenuated, VEGF signaling.

Given the critical role of VEGF signaling in development, it is reasonable to conclude that the presence of ESDN is not an absolute requirement for VEGF signaling; rather, ESDN appears to be involved in the fine-tuning of VEGF responses. It is also possible that during development, there are compensatory mechanisms that ultimately overcome the absence of ESDN, and these mechanisms are less functional in adulthood. Inhibition of VEGF signaling is an established approach to treating age-related macular degeneration and tumor angiogenesis and may prove to be useful for other disorders where VEGF plays a key role. On the other hand, enhancing VEGF responses to promote angiogenesis is a promising approach to treating chronic ischemia. Regulation of 
A

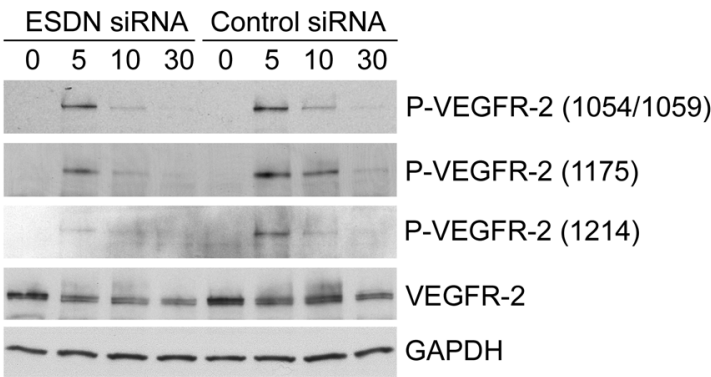

B

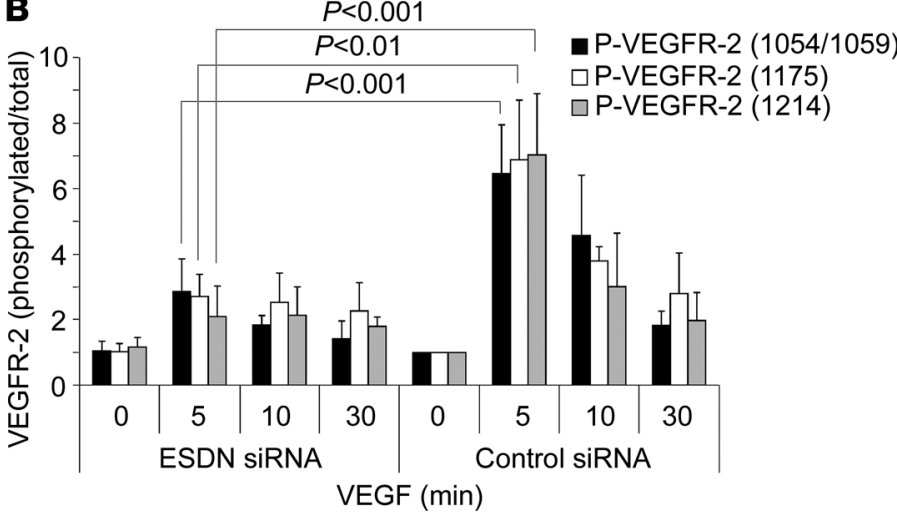

C

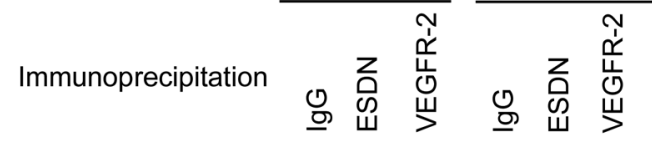

VEGFR-2

ESDN
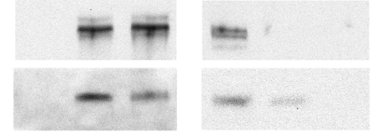

D

WT Esdn ${ }^{-/-}$

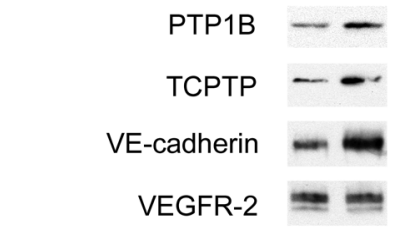

Immunoprecipitation $\overline{\text { VEGFR-2 }}$

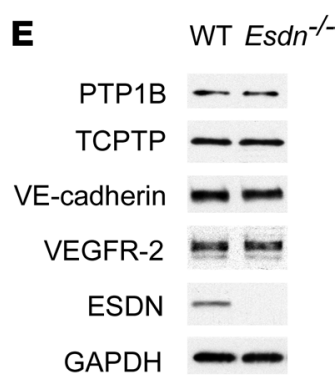

Figure 7

ESDN and VEGFR-2 phosphorylation and complex formation in ECs. (A and B) Western blot example and quantification of VEGF-induced VEGFR-2 phosphorylation in HUVECs following siRNA-mediated ESDN downregulation. (C) ESDN-VEGFR-2 complex formation. VEGFR-2 or ESDN immunoprecipitation from HUVECs, followed by Western blot analysis of ESDN and VEGFR-2 in the precipitate and the remaining supernatant. (D) PTP1B, TCPTP, and VE-cadherin co-immunoprecipitation with VEGFR-2 in Esdn $n^{-1}$ and WT MLECs. VEGFR-2 immunoprecipitation was followed by Western blot analysis for PTP1B, TCPTP, and VE-cadherin. (E) Western blot analysis of PTP1B, TCPTP, VE-cadherin, and VEGFR-2 expression in WT and Esdn ${ }^{-1-}$ MLECs.

VEGF signaling by ESDN raises the possibility that ESDN may serve as a novel therapeutic target for a wide variety of disorders, including those associated with impaired angiogenesis.

\section{Methods}

Reagents. Reagents were purchased from Sigma-Aldrich, unless stated otherwise. Human recombinant VEGF was obtained from the National Cancer Institute. $\left[{ }^{3} \mathrm{H}\right]$-thymidine was obtained from PerkinElmer. The polyclonal rabbit anti-ESDN antibody and monoclonal mouse anti-human and -murine $\alpha$-SMA were obtained from Sigma-Aldrich. Phycoerythrin (PE)conjugated polyclonal sheep anti-ESDN antibody was purchased from R\&D Systems. The antibodies for phospho-MAPK p44/42 (Thr202/ Tyr204), total MAPK p44/42, phospho-p38 (Thr180/Tyr182), total p38, phospho-Akt (Ser473), total Akt, phospho-VEGFR-2 (Tyr1175), total VEGFR-2, and phospho-eNOS (Ser1177) were obtained from Cell Signaling Technologies. Anti-total eNOS, anti-TCPTP, anti-VE-cadherin, and anti-human and -mouse PDGFR- $\beta$ antibodies were obtained from Santa Cruz Biotechnology Inc. Anti-phospho-VEGFR-2 (Tyr1054/Tyr1059), anti-phospho-VEGFR-2 (Tyr1214), and sheep anti-rat IgG Dynabeads were obtained from Invitrogen Life Technologies. Rat anti-mouse CD31 antibody was obtained from BD Biosciences. Anti-NG2 antibody was obtained from Millipore. The anti-PTP1B antibody was obtained from Abcam. The anti-CD68 antibody was obtained from Cedarlane.

Cell culture. Single-donor HUVECs were isolated and cultured on gelatin-coated plates in medium 199 supplemented with $20 \%$ heat-inactivated FBS, endothelial cell growth supplement (ECGS), glutamine, and penicil- lin-streptomycin as described previously (41). Subcultures were obtained by trypsinization and used at up to passage 5. MLECs were isolated from 4-week-old WT and Esdn-null mice by two rounds of immunoselection with CD31-conjugated magnetic beads according to a previously described procedure (42). The purity of MLEC isolations, as assessed by flow cytometry using CD31 as a marker of ECs, was consistently $>95 \%$. Furthermore, there was no difference in cell purity between KO and WT cells. The cells were cultured and maintained in medium 199 containing 20\% FBS and ECGS. The remaining cells had a fibroblast-like morphology and were cultured as fibroblasts in medium 199 containing 20\% FBS. Human and vascular smooth muscle cells were isolated and maintained in culture as described previously (38). The experiments were performed with cells at early confluence, unless specified otherwise.

DNA constructs and retroviral transduction. HA-tagged human ESDN and enhanced GFP retroviral expression were performed as described previously (12). HUVECs were transduced by 6 drug-free serial infections. Approximately $90 \%$ of the cells expressed the transduced proteins.

RNA interference. Three 25-bp duplex siRNAs that specifically target different regions of human ESDN mRNA (NM_080927) were designed and synthesized by Invitrogen Life Technologies: ESDN2245: 5'-GGA AUUGUUGGUACACUUCAUCAAA; ESDN769: 5'-GGAAUUGGAGUCAGCAGAACUGAAA; ESDN1081: 5'-GGUGUGCAUGCAGGAGUAGUGUCAA. The cells were transfected with either ESDN siRNA or a universal negative control siRNA (Invitrogen, cat. 12935200) using FuGENE 6 Transfection Reagent (Roche Diagnostics) according to the manufacturer's instructions. To downregulate phosphatase expression, MLECs were trans- 
A

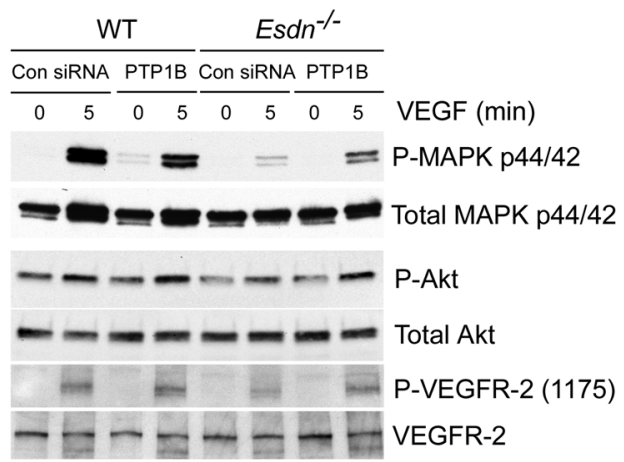

B

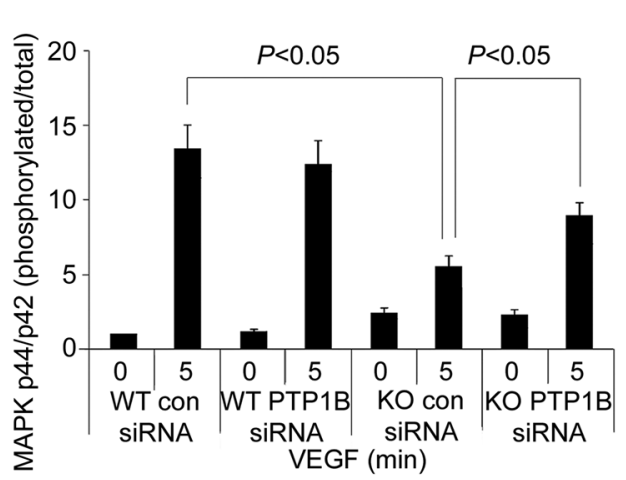

C
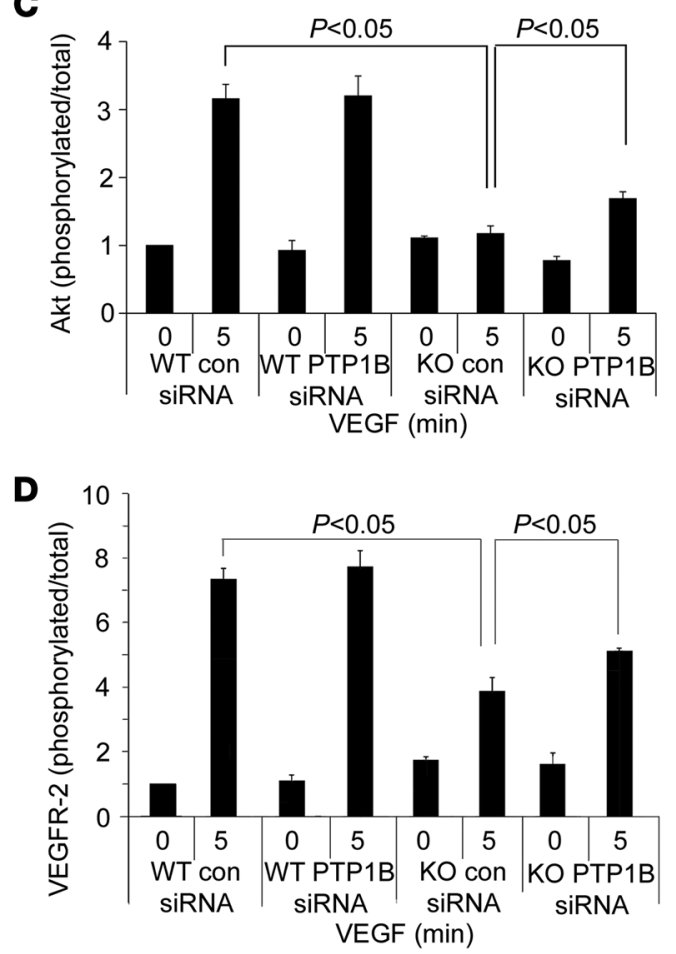

E

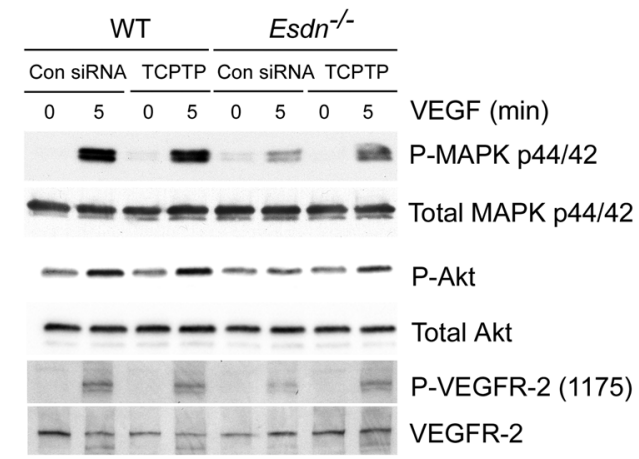

$\mathbf{F}$

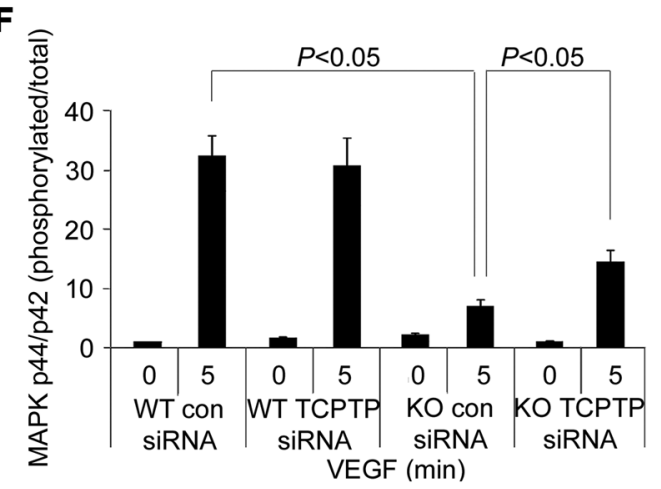

G

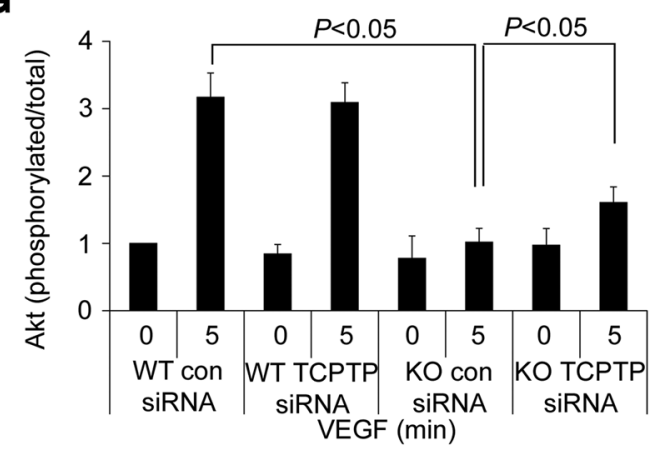

H

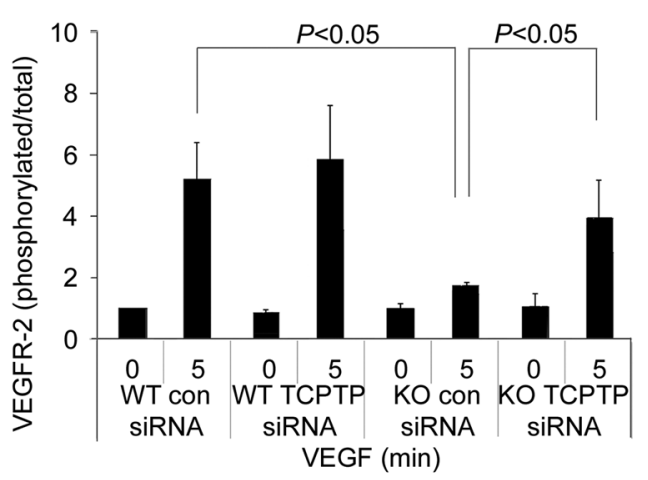

Figure 8

Protein tyrosine phosphatase downregulation and VEGF signaling in MLECs. (A-H) Western blot analysis of VEGF-induced MAPK p44/42 Tyr202/ Tyr204, Akt Ser473, and VEGFR-2 Tyr1175 phosphorylation following siRNA-mediated PTP1B (A) or TCPTP (E) downregulation in Esdn ${ }^{-1-}$ (KO) and WT MLECs. Quantitative analysis of the ratio of phosphorylated to total MAPK and VEGFR-2 from three independent experiments is shown in $\mathbf{B}-\mathbf{D}$ and $\mathbf{F}-\mathbf{H}$. 


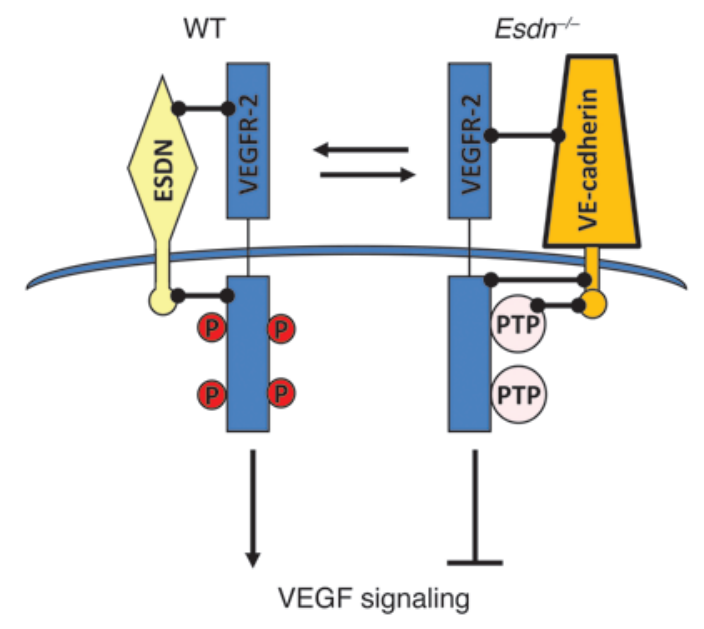

Figure 9

Schematic representation of the ESDN effect on VEGF signaling in endothelial cells. ESDN associates with VEGFR-2 and inhibits PTP1B, TCPTP, and VE-cadherin association with VEGFR-2, thus promoting VEGF-induced VEGFR-2 phosphorylation and downstream signaling. In the absence of ESDN, enhanced VEGFR-2-phosphatase (and VEcadherin) association attenuates VEGF signaling in ECs.

fected with target-specific pooled siRNAs for PTP1B and/or TCPTP or a control siRNA (Santa Cruz Biotechnology Inc.) according to the manufacturer's instructions. Forty-eight hours later, the MLECs were serum starved for 24 hours and treated with VEGF as indicated.

Immunofluorescence staining. Immunofluorescence staining was performed using standard techniques on acetone-fixed EC monolayers or 5 - $\mu$ m-thick acetone-fixed cryostat sections. Primary antibodies included rabbit anti-human ESDN (Sigma-Aldrich), rat anti-HA (Roche Applies Science), rat anti-mouse CD31 (BD Biosciences - Pharmingen), and isotypematched controls. Nuclei were detected by DAPI (Invitrogen).

Quantitative RT-PCR. Total mRNA isolated using QIAGEN kits was reverse transcribed. Real-time PCR was performed in triplicate using this cDNA with the QuantiTect SYBR Green PCR kit (QIAGEN) or Taqman gene assays (Applied Biosystems) according to the manufacturer's instructions. The results were normalized to GAPDH (for cells) or 18S rRNA (for tissues). The following primers, synthesized at the Yale University Keck Facility, were used: human ESDN: 5'-GGCCCTGAGAGTGGAACCCTTACAT- $3^{\prime}$ and $5^{\prime}$-TTCATTTGCAACCCCAGACCAC-3', human VEGFR1: 5'-ATCATTCCGAAGCAAGGTGTG-3' and 5'-AAACCCATTTGGCA-

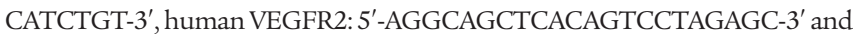
5'-GTCTTTTCCTGGGGCACCTTCTA-3', human GAPDH: 5'-CCAAGGTCATCCATGACAAC- $3^{\prime}$ and $5^{\prime}$-TGTCATACCAGGAAATGAGC- $3^{\prime}$, zebrafish $d c b l d 2:$ 5'-AGAGATTCTTCTCCTCTGTG-3' and 5'-AGGCACAGATGTCACATTGT-3', zebrafish $\alpha$-actin (actb1): $5^{\prime}$-ATGGAGAAGATCTGGCATC-3' and 5'-CACCAGAGTCCATCACAAT-3', mouse Esdn exon 2-3: 5'-GACGGGAGAAAGGATTCGCA-3' and 5'- GAAGCCAAAAATCCTCGCCC- ${ }^{\prime}$, mouse Esdn exon 15-16: 5'-GTGGAAAGGAATGAAGCAGC-3' and 5'-ATACATTCTACATCACTACCACTAAG-3'. In addition, Hs00294635_ml, Hs00911706_m1, Hs01052936_m1, and Hs02758991_m1 Taqman primer sets were respectively used for human ESDN, VEGFR2, VEGFR-1, and GAPDH in a subset of studies. For murine Esdn, Cd31, Ptp1b, Tcptp, VE-cadherin, Vegfr2, Vegfr1, Gapdh, and 18s rRNA, the Mm00472304_m1, Mm00476702_ m1, Mm00448427_m1, Mm00501226_m1 and Mm00486938_m1, Mm01222421_m1, Mm00438980_m1, and Mm99999915_g1 Taqman primer sets and the 18S rRNA Taqman assay reagent were used.
Cell growth assay. ECs expressing ESDN-HA or GFP or ECs transfected with ESDN or control siRNA for 24 hours were seeded at a density of 2,500 cells/well into 96-well plates. Twenty-four hours later, the medium was replaced with medium 199 containing $1 \%$ FBS. The next day, the cells were stimulated in multiple wells $(n=16)$ with VEGF $(50 \mathrm{ng} / \mathrm{ml})$ or control buffer for 4 days and the cell numbers were tracked by MTT assay (Millipore) according to the manufacturer's recommendations.

$\left[{ }^{3} \mathrm{H}\right]$-thymidine incorporation assay. DNA synthesis was quantified by measuring $\left[{ }^{3} \mathrm{H}\right]$-thymidine incorporation. Ten thousand HUVECs transduced with ESDN or GFP retrovirus, HUVECs transfected with either ESDN or

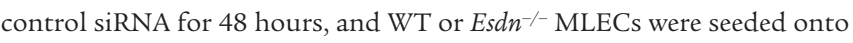
24-well culture plates. Twenty-four hours later, the medium was replaced with medium 199 containing $1 \%$ FBS. The next day, the cells were treated with VEGF $(50 \mathrm{ng} / \mathrm{ml})$ or control buffer in medium 199 containing $1 \%$ FBS in triplicate wells. After 18 hours, $2 \mu \mathrm{Ci}\left[{ }^{3} \mathrm{H}\right]$-thymidine (0.074 MBq; GE Healthcare) was added to each well. Six hours later, the cells were rinsed and fixed in ice-cold methanol, and DNA was precipitated by $5 \%$ trichloroacetic acid and recovered with $\mathrm{NaOH}(0.3 \mathrm{~N})$ at room temperature. Aliquots were assayed for $\left[{ }^{3} \mathrm{H}\right]$-thymidine incorporation by liquid scintillation counting (Beckman Coulter). The counts were normalized to the control sample and expressed as percent increase in $\left[{ }^{3} \mathrm{H}\right]$-thymidine incorporation over non-stimulated cells.

Migration assays. The modified Boyden chamber migration assay was performed as described previously (42). Briefly, the undersurface of polycarbonate filters ( $8-\mu \mathrm{m}$ pore size, Millipore) was coated with fibronectin $(20 \mu \mathrm{g} / \mathrm{ml})$ in PBS. Medium 199 with $0.5 \%$ bovine serum albumin containing VEGF $(50 \mathrm{ng} / \mathrm{ml})$ or control buffer was added in the lower well. ECs expressing ESDN-HA or GFP, HUVECs transfected with ESDN or control siRNA for 72 hours, or $E s d n^{-/-}$or WT MLECs were maintained in ECGSfree medium 199 with $1 \%$ FBS overnight, and the next day, $1 \times 10^{5}$ ECs were loaded into each upper chamber in triplicate wells and cultured for 6 hours at $37^{\circ} \mathrm{C}$ in the presence of $5 \% \mathrm{CO}_{2}$ in a humidified incubator. After removing non-migrating cells using cotton swabs, the transmigrated cells on the lower surfaces of the filters were counted microscopically following staining with $0.2 \%$ crystal violet in $10 \%$ ethanol. The data were expressed as percent increase in the number of transmigrated cells in the presence of VEGF.

Immunoblotting. ECs expressing ESDN-HA or GFP or ECs transfected with ESDN or control siRNA for 72 hours were placed in medium 199 with $1 \%$ FBS for 24 hours, followed by stimulation with VEGF $(50 \mathrm{ng} / \mathrm{ml})$ or control buffer for the indicated time. MLECs from WT and Esdn-null mice were serum starved in FBS-free and ECGS-free medium 199 for 24 hours prior to stimulation with VEGF or control buffer. The cells were washed and lysed in ice-cold RIPA buffer $(150 \mathrm{mM} \mathrm{NaCl}, 50 \mathrm{mM}$ Tris-HCl, $\mathrm{pH}$ 8.0, 1.0\% NP-40, 0.5\% sodium deoxycholate, and 0.1\% SDS) supplemented with complete proteinase inhibitor (Roche Applied Sciences) and phosphatase inhibitor cocktails (Sigma-Aldrich). The cell homogenates were centrifuged $\left(13,000 \mathrm{~g}, 15\right.$ minutes, $\left.4^{\circ} \mathrm{C}\right)$, the supernatants were collected, and protein concentrations were determined (DC Protein Assay Reagent, Bio-Rad). Equal amounts of protein were resolved by SDS-PAGE and transferred electrophoretically onto an Immun-Blot PVDF membrane (Bio-Rad). The membranes were probed with primary antibodies, followed by horseradish peroxidase-conjugated secondary antibodies (Zymed, Life Technologies) and developed using a chemiluminescence detection system (PerkinElmer). For the phosphorylated antibodies, the membranes were stripped using Restore Western blot strip buffer (Pierce) and reprobed with total antibodies. GAPDH was used as loading control. The films were scanned and quantitated using Kodak 1D 3.5 software.

Immunoprecipitation. Cell lysates (containing $500 \mu \mathrm{g}$ of protein) were incubated with $1.5 \mathrm{mg}$ protein A Dynabeads (Invitrogen) for 1 hour at $4^{\circ} \mathrm{C}$, after which the beads were removed by centrifugation to clear the 
lysate. The precleared lysates were incubated with the respective antibodies bound to protein A Dynabeads for 1 hour at $4{ }^{\circ} \mathrm{C}$, then washed with lysis buffer (150 mM NaCl, $50 \mathrm{mM}$ Tris-HCl, pH 8.0, 1.0\% NP-40, 0.5\% sodium deoxycholate, and $0.1 \%$ SDS supplemented with complete proteinase inhibitor, Roche Applied Sciences). The beads were separated on a magnet and the immunocomplexes were resuspended in $20 \mu \mathrm{l}$ SDS-PAGE sample buffer for further analysis.

Targeted inactivation of the Esdn gene. An RPCI-22 129/SvEvTACBr mouse bacterial artificial chromosome library (Center for Applied Genomics) was screened using an Esdn cDNA probe to identify genomic clones containing the putative promoter and exon 1 of the Esdn gene (43). The targeting vector was constructed by cloning a 6,428-bp oligonucleotide fragment spanning Esdn's putative promoter and exon 1 between the first two loxP sites of pEASYflox. The construct contains a neomycin cassette (Neo), flanked by two loxP sites downstream of the Esdn cloning site. The linearized targeting construct was electroporated into TC-1 embryonic stem cells (derived from 129/SvEvTac). The targeted clones were selected with G418 and ganciclovir. Resistant clones were screened for homologous recombination by PCR and confirmed by Southern blotting. A clone with a normal karyotype was selected for injection into blastocysts derived from black C57BL/6J mice to generate chimeras. Germline transmission was confirmed by mating with wild-type C57BL/6J females. These mice were mated with MeuCre 40 mice to mediate recombination in vivo, resulting in complete deletion of the Esdn putative promoter and exon 1, as well as the Neo gene (44). Es $\mathrm{dn}^{-/-}$mice were obtained by mating male and female heterozygous $\left(E s d n^{+-}\right)$mice. Esdn deletion was confirmed by genotyping, Southern blot analysis, RT-PCR, Northern blot analysis, Western blot analysis, and immunostaining. These mice were backcrossed to the WT C57BL/6J strain for ten generations. Eight- to 12 -week-old $\mathrm{F}_{3}$ to $\mathrm{F}_{10}$ generation knockout mice and their WT littermates of the same sex were used in this study. To generate endothelial-specific conditional knockout mice, transgenic mice expressing Cre recombinase under the control of the VE-cadherin promoter (B6.Cg$\mathrm{Tg}$ (Cdh5-cre)7Mlia/J, Jackson Laboratory) (15) and heterozygous for Esdn (VE-cadherin $\left.\mathrm{Cre}^{+/-}, E s d n^{+/-}\right)$were crossed with Esdn $n^{f l f l}$ animals. Esdn gene deletion, confirmed by Western blotting, was detected at variable degrees in different animals.

En face staining. WT or Esdn $n^{-/-}$mice were perfused with 4\% paraformaldehyde to fix ECs. After removal of the fatty tissues, the aorta was mounted on a slide with the lumen opened and facing up. The cells were permeabilized with $0.1 \%$ Triton X-100 in $1 \times$ PBS 3 times for 5 minutes each and the en face staining protocol was performed as described previously (45).

Murine lung in vivo eNOS phosphorylation assay. In vivo eNOS phosphorylation was performed as described previously with minor modifications (46). WT and Esdn knockout male mice (8-12 weeks old) were anesthetized, and a catheter was introduced into the left jugular vein for administration of VEGF. Either human VEGF $165(20 \mu \mathrm{g})$ or saline was injected and allowed to circulate for 5 minutes. The animal was then perfused with $5 \mathrm{ml}$ PBS supplemented with phosphatase inhibitors, after which the lungs were harvested for Western blot analysis.

Ear angiogenesis assay. Ear angiogenesis studies were performed as described previously with minor modifications (47). Adenoviruses encoding murine VEGF- $\mathrm{A}_{164}$ or LacZ $\left(9 \times 10^{8} \mathrm{PFU}\right.$ in $15 \mu \mathrm{l}$, a gift from William Sessa, Yale University) were injected intradermally into the opposite ears of $E s d n^{-/}$mice and their WT littermates. After 5 days, the animals were euthanized and the ears were harvested. Immunostaining was performed on three $5-\mu \mathrm{m}$ sections, each separated by $500 \mu \mathrm{m}$, starting from the injection site. Similarly, for each ear the RNA was isolated from three $100-\mu \mathrm{m}$-long tissue segments, each separated by $500 \mu \mathrm{m}$, and the results were averaged. The positive staining area was quantified using ImageJ software (http://rsbweb.nih.gov/ij/) and expressed as an angiogenesis score representing the percent increase in the relative area of positive staining in VEGF-treated ears compared with control, LacZ-treated ears.

Matrigel angiogenesis assay. Growth factor-reduced Matrigel (0.5 ml, BD Biosciences) with or without VEGF $(2 \mu \mathrm{g} / \mathrm{ml})$ was subcutaneously injected into opposite iliac regions of WT and $E s d n^{-/-}$mice, where it rapidly solidified. After 5 days, the animals were euthanized and the plugs were harvested for immunostaining. Quantification of angiogenesis was performed as described above on three sections per plug to define the fraction of positive area for each section. The data were expressed as percent increase in the relative area of positive staining in plugs enriched with VEGF compared with those without VEGF.

Retinal angiogenesis assay. Eyes from postnatal day 5 and 10 littermates were fixed in $4 \%$ paraformaldehyde for 30 minutes. Retinas were dissected and rinsed in PBS, then permeabilized overnight in PBS containing 1\% BSA (Roche Applied Sciences) and 0.5\% Triton X-100. The permeabilized retinas were incubated with biotin-conjugated isolectin from Bandeiraea simplicifolia (20 $\mu \mathrm{g} / \mathrm{ml}$, Sigma-Aldrich), followed by Alexa Fluor 488-conjugated streptavidin (Invitrogen Life Technologies), and whole mounts were prepared for photography under a fluorescence microscope. The total length and number of branch points of isolectin-positive vessels in the entire retina were quantified on composite high-magnification images using Biologic CMM Analyzer software (College of France).

Hindlimb ischemia model. The femoral artery of Es $d n^{-/-}$and WT mice (8-12 weeks) was ligated at two positions spaced $5 \mathrm{~mm}$ apart, one just proximal to the groin ligament, and the second distal to it and proximal to the popliteal artery. The branches between the two positions were ligated and the arterial segment between the ligatures was excised. Laser-Doppler flow imaging was carried out using a Moor Infrared Laser Doppler Imager (LDI; Moor Instruments) at $37 \pm 0.5^{\circ} \mathrm{C}$ under anesthesia at different time points before and aftersurgery. The data were analyzed with Moor LDI image processing software V3.09 and reported as the ratio of flow in the ligated/control hindlimb. In another group of animals, the calf muscles were harvested under anesthesia at 2 weeks after proximal femoral artery ligation and placed in Tissue-Tek OCT compound (Sakura Finetek USA Inc.), and three random 10- $\mu \mathrm{m}$ frozen sections of the gastrocnemius muscle per animal were used for CD31 staining. The capillary density was quantified by measuring the CD31-positive area relative to the muscle area using ImageJ software and the data were expressed as percent increase in the relative area of CD31-positive staining in the ligation side compared with the opposite hindlimb.

Micro-CT angiography. Two weeks after femoral ligation, Es $d n^{-/-}$and WT mice were anesthetized and a vascular contrast agent was injected. The preparation, acquisition, and analysis of micro-CT images were carried out as described previously (4).

Southern blotting. Genomic DNA was extracted from the tails of Es $d n^{-/-}$ mice and their WT littermates using lysis buffer $(100 \mathrm{mM} \mathrm{NaCl}, 20 \mathrm{mM}$ Tris-HCl, pH 7.6, $10 \mathrm{mM}$ EDTA, $0.5 \% \mathrm{SDS}$, and $0.5 \mathrm{mg} / \mathrm{ml}$ proteinase $\mathrm{K}$ ). A total of $20 \mu \mathrm{g}$ of DNA was digested with Avr II and then separated by electrophoresis. After acid depurination, alkaline denaturation, and neutralization, the DNA was transferred to a Genomic Tested Blotting Membrane (Bio-Rad). The DNA was then fixed to the membrane using a UV crosslinker (Stratagene). The probe was generated by Not I digestion of the targeting vector and ${ }^{32} \mathrm{P}$-labeled using the Random Prime Labeling System (Amersham Biosciences). The membrane was probed at $65^{\circ} \mathrm{C}$, and the signal was detected by exposing the membranes to Kodak Biomax MR film (Carestream Health).

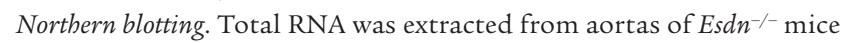
and their WT littermates using the RNeasy kit (QIAGEN). A total of $15 \mu \mathrm{g}$ of RNA was electrophoresed on a $1 \%$ agarose-formaldehyde gel, transferred to a Hybond-N Transfer Membrane (Amersham Biosciences), 
and fixed to the membrane using a UV crosslinker. The membrane was probed at $42^{\circ} \mathrm{C}$ with ${ }^{32} \mathrm{P}$-labeled Es $d n$ probes generated by RT-PCR encompassing exons $2-3$ and $15-16$, and GAPDH. The signals were detected by autoradiography.

Zebrafish maintenance, cell sorting, and MO knockdown. $\mathrm{Tg}$ (fli1:nEGFP); $\mathrm{Tg}(\mathrm{kdrl}: \mathrm{mCherry})$ zebrafish were maintained under standard laboratory conditions. Standard control or gene-specific MOs targeting the ESDN splice site between exon 2 and intron 2 (sp2, AGTTCATTACTGTTTCTCACCAATT) or intron 1 and exon 2 (sp1, AGCCGTCCCCTGAAAAACACCCAGA; Gene Tools LLC) were used to achieve knockdown of ESDN. To assess vascular development, one- to four-cell embryos were injected with $0.2 \mathrm{pmol}$ of MO using a Picospritzer (Parker). The embryos were scored for GFP/mCherry expression at 28-32 hours after fertilization using a Leica M205 FA with a Roper CoolSnap Camera. The ISVs were categorized into two groups based on whether they were anastomosed to DLAV. The number of ISVs connected to DLAV per embryo was determined. Endothelial cells were isolated from $\mathrm{Tg}(\mathrm{kdrl}$ :EGFP) transgenic zebrafish embryos using flow cytometry, as previously reported (48). After flow cytometry, $\mathrm{EGFP}^{+}$endothelial cells and EGFP- cells (presumed non-endothelial cells) were lysed to isolate RNA samples, which were subsequently used for real-time PCR analyses for the dcbld2 transcript.

Flow cytometry. Cells harvested using trypsin-free cell dissociation medium were incubated with PE-conjugated anti-human or anti-mouse ESDN, VEGFR-1, or R-2 (R\&D Systems) or control antibodies. A total of 5,000 cells were analyzed per sample on a FACSCalibur (BD), as previously described (41).

Statistics. All values are expressed as mean \pm SEM. The significance of the results was assessed by 2 -tailed ratio $t$ test (for paired non-parametric values) or 2-way ANOVA with Bonferroni post hoc test (for $>2$ groups). A $P$ value less than 0.05 was considered significant.

Study approval. All studies were performed under protocols approved by Yale University and Veterans Affairs Connecticut Healthcare System Institutional Animal Care and Use and Human Investigational Committees.

\section{Acknowledgments}

This work was supported by NIH grants R01-HL114703, R01HL112992, R01-HL085093, and Department of Veterans Affairs grant I01-BX001750.

Received for publication November 8, 2012, and accepted in revised form August 29, 2013.

Address correspondence to: Mehran M. Sadeghi, VA Connecticut Healthcare System, 950 Campbell Avenue, 111B, West Haven, Connecticut 06516, USA. Phone: 203.932.5711, ext. 3398; Fax: 203.937.3884; E-mail: mehran.sadeghi@yale.edu.

Leila Esmailzadeh's present address is: Boehringer Ingelheim, Ridgefield, Connecticut, USA.

Abolfazl Asadi's present address is: Stockholm University, Stockholm, Sweden.

Mark Collinge's present address is: Pfizer, Groton, Connecticut, USA.

Xuan Li's present address is: Nanjing University of Chinese Medicine, Nanjing, China.
1. Koch S, Tugues S, Li X, Gualandi L, Claesson-Welsh L. Signal transduction by vascular endothelial growth factor receptors. Biochem J. 2011;437(2):169-183.

2. Simons M. An inside view: VEGF receptor trafficking and signaling. Physiology (Bethesda). 2012; 27(4):213-222.

3. Sawamiphak S, et al. Ephrin-B2 regulates VEGFR2 function in developmental and tumour angiogenesis. Nature. 2010;465(7297):487-491.

4. Lanahan AA, et al. VEGF receptor 2 endocytic trafficking regulates arterial morphogenesis. Dev Cell. 2010;18(5):713-724.

5. Fantin A, Maden CH, Ruhrberg C. Neuropilin ligands in vascular and neuronal patterning. Biochem Soc Trans. 2009;37(pt 6):1228-1232.

6. Pellet-Many C, Frankel P, Jia H, Zachary I. Neuropilins: structure, function and role in disease. Biochem J. 2008;411(2):211-226.

7. Gu C, et al. Neuropilin-1 conveys semaphorin and VEGF signaling during neural and cardiovascular development. Dev Cell. 2003;5(1):45-57.

8. Prahst C, et al. Neuropilin-1-VEGFR-2 complexing requires the $\mathrm{PDZ}$-binding domain of neuropilin-1. J Biol Chem. 2008;283(37):25110-25114.

9. Salikhova A, et al. Vascular endothelial growth factor and semaphorin induce neuropilin-1 endocytosis via separate pathways. Circ Res. 2008;103(6):e71-e79.

10. Kobuke K, et al. ESDN, a novel neuropilin-like membrane protein cloned from vascular cells with the longest secretory signal sequence among eukaryotes, is up-regulated after vascular injury. J Biol Chem. 2001;276(36):34105-34114.

11. Koshikawa K, et al. Significant up-regulation of a novel gene, CLCP1, in a highly metastatic lung cancer subline as well as in lung cancers in vivo. Oncogene. 2002;21(18):2822-2828.

12. Sadeghi MM, et al. ESDN is a marker of vascular remodeling and regulator of cell proliferation in graft arteriosclerosis. Am J Transplant.
2007;7(9):2098-2105.

13. Yu J, et al. Endothelial nitric oxide synthase is critical for ischemic remodeling, mural cell recruitment, and blood flow reserve. Proc Natl Acad Sci U S A. 2005;102(31):10999-11004.

14. Gerhardt $H$, et al. VEGF guides angiogenic sprouting utilizing endothelial tip cell filopodia. $J$ Cell Biol. 2003;161(6):1163-1177.

15. Alva JA, et al. VE-Cadherin-Cre-recombinase transgenic mouse: a tool for lineage analysis and gene deletion in endothelial cells. Dev Dyn. 2006;235(3):759-767.

16. Georgijevic S, Subramanian Y, Rollins EL, StarovicSubota O, Tang AC, Childs SJ. Spatiotemporal expression of smooth muscle markers in developing zebrafish gut. Dev Dyn. 2007;236(6):1623-1632.

17. Santoro MM, Pesce G, Stainier DY. Characterization of vascular mural cells during zebrafish development. Mech Dev. 2009;126(8-9):638-649.

18. Eilken HM, Adams RH. Dynamics of endothelial cell behavior in sprouting angiogenesis. Curr Opin Cell Biol. 2010;22(5):617-625.

19. Potente M, Gerhardt H, Carmeliet P. Basic and therapeutic aspects of angiogenesis. Cell. 2011; 146(6):873-887.

20. Olsson AK, Dimberg A, Kreuger J, Claesson-Welsh L. VEGF receptor signalling - in control of vascular function. Nat Rev Mol Cell Biol. 2006;7(5):359-371.

21. Nakamura $Y$, et al. Role of protein tyrosine phosphatase $1 \mathrm{~B}$ in vascular endothelial growth factor signaling and cell-cell adhesions in endothelial cells. Circ Res. 2008;102(10):1182-1191.

22. Mattila E, Auvinen K, Salmi M, Ivaska J. The protein tyrosine phosphatase TCPTP controls VEGFR2 signalling. J Cell Sci. 2008;121(pt 21):3570-3580.

23. Baumer $S$, et al. Vascular endothelial cell-specific phosphotyrosine phosphatase (VE-PTP) activity is required for blood vessel development. Blood. 2006;107(12):4754-4762.
24. Mellberg S, et al. Transcriptional profiling reveals a critical role for tyrosine phosphatase VE-PTP in regulation of VEGFR2 activity and endothelial cell morphogenesis. FASEB J. 2009;23(5):1490-1502.

25. Nakayama $M$, et al. Spatial regulation of VEGF receptor endocytosis in angiogenesis. Nat Cell Biol. 2013;15(3):249-260.

26. Grazia Lampugnani $M$, et al. Contact inhibition of VEGF-induced proliferation requires vascular endothelial cadherin, beta-catenin, and the phosphatase DEP-1/CD148. J Cell Biol. 2003;161(4):793-804.

27. Chabot C, Spring K, Gratton JP, Elchebly M, Royal I. New role for the protein tyrosine phosphatase DEP-1 in Akt activation and endothelial cell survival. Mol Cell Biol. 2009;29(1):241-253.

28. Guo DQ, et al. Tumor necrosis factor employs a protein-tyrosine phosphatase to inhibit activation of KDR and vascular endothelial cell growth factorinduced endothelial cell proliferation. J Biol Chem. 2000;275(15):11216-11221.

29. Gallicchio M, et al. Inhibition of vascular endothelial growth factor receptor 2-mediated endothelial cell activation by Axl tyrosine kinase receptor. Blood. 2005;105(5):1970-1976.

30. Lanahan A, et al. The neuropilin 1 cytoplasmic domain is required for VEGF-A-dependent arteriogenesis. Dev Cell. 2013;25(2):156-168.

31. Kolodkin AL, Levengood DV, Rowe EG, Tai YT, Giger RJ, Ginty DD. Neuropilin is a semaphorin III receptor. Cell. 1997;90(4):753-762.

32. Neufeld G, Cohen T, Shraga N, Lange T, Kessler O, Herzog Y. The neuropilins: multifunctional semaphorin and VEGF receptors that modulate axon guidance and angiogenesis. Trends Cardiovasc Med. 2002;12(1):13-19.

33. Lampugnani MG, Orsenigo F, Gagliani MC, Tacchetti C, Dejana E. Vascular endothelial cadherin controls VEGFR-2 internalization and signal- 
ing from intracellular compartments. J Cell Biol. 2006;174(4):593-604

34. Soker S, Takashima S, Miao HQ, Neufeld G, Klagsbrun M. Neuropilin-1 is expressed by endothelial and tumor cells as an isoform-specific receptor for vascular endothelial growth factor. Cell. 1998;92(6):735-745.

35. Stuible M, Tremblay ML. In control at the ER: PTP1B and the down-regulation of RTKs by dephosphorylation and endocytosis. Trends Cell Biol. 2010;20(11):672-679.

36. Manickam V, et al. Regulation of vascular endothelial growth factor receptor 2 trafficking and angiogenesis by Golgi localized t-SNARE syntaxin 6. Blood. 2010;117(4):1425-1435.

37. Miyake S, Lupher ML Jr, Druker B, Band H. The tyrosine kinase regulator $\mathrm{Cbl}$ enhances the ubiquitination and degradation of the platelet-derived growth factor receptor alpha. Proc Natl Acad Sci US A. 1998;95(14):7927-7932.

38. Guo X, Nie L, Esmailzadeh L, Zhang J, Bender JR, Sadeghi MM. Endothelial and smooth muscle- derived neuropilin-like protein regulates plateletderived growth factor signaling in human vascular smooth muscle cells by modulating receptor ubiquitination. J Biol Chem. 2009;284(43):29376-29382.

39. Duval M, Bedard-Goulet S, Delisle C, Gratton JP. Vascular endothelial growth factor-dependent down-regulation of Flk-1/KDR involves Cbl-mediated ubiquitination. Consequences on nitric oxide production from endothelial cells. J Biol Chem. 2003;278(22):20091-20097.

40. Rahimi N. A role for protein ubiquitination in VEGFR-2 signalling and angiogenesis. Biochem Soc Trans. 2009;37(pt 6):1189-1192.

41. Sadeghi MM, et al. Detection of injury-induced vascular remodeling by targeting activated alphavbeta3 integrin in vivo. Circulation. 2004;110(1):84-90.

42. Zhang $\mathrm{H}$, et al. AIP1 functions as an endogenous inhibitor of VEGFR2-mediated signaling and inflammatory angiogenesis in mice. J Clin Invest. 2008;118(12):3904-3916.

43. Auerbach W, et al. Establishment and chimera analysis of $129 / \mathrm{SvEv}$ - and C57BL/6-derived mouse embryonic stem cell lines. Biotechniques. 2000;29(5):1024-1028.

44. Leneuve $P$, et al. Cre-mediated germline mosaicism: a new transgenic mouse for the selective removal of residual markers from tri-lox conditional alleles. Nucleic Acids Res. 2003;31(5):e21.

45. Zeng L, et al. Sustained activation of XBP1 splicing leads to endothelial apoptosis and atherosclerosis development in response to disturbed flow. Proc Natl Acad Sci U S A. 2009;106(20):8326-8331.

46. Smith NR, et al. Acute pharmacodynamic and antivascular effects of the vascular endothelial growth factor signaling inhibitor AZD2171 in Calu-6 human lung tumor xenografts. Mol Cancer Ther. 2007;6(8):2198-2208.

47. Ackah E, et al. Akt1/protein kinase Balpha is critical for ischemic and VEGF-mediated angiogenesis. J Clin Invest. 2005;115(8):2119-2127.

48. Schmitt CE, Woolls MJ, Jin SW. Mutant-specific gene expression profiling identifies SRY-related HMG box $11 \mathrm{~b}$ (SOX11b) as a novel regulator of vascular development in zebrafish. Mol Cells. 2013;35(2):166-172. 